\title{
CONTROL PROBLEMS FOR CONVECTION-DIFFUSION EQUATIONS WITH CONTROL LOCALIZED ON MANIFOLDS
}

\author{
Phuong Anh Nguren ${ }^{1}$ And Jean-Pierre Raymond ${ }^{1}$
}

\begin{abstract}
We consider optimal control problems for convection-diffusion equations with a pointwise control or a control localized on a smooth manifold. We prove optimality conditions for the control variable and for the position of the control. We do not suppose that the coefficient of the convection term is regular or bounded, we only suppose that it has the regularity of strong solutions of the Navier-Stokes equations. We consider functionals with an observation on the gradient of the state. To obtain optimality conditions we have to prove that the trace of the adjoint state on the control manifold belongs to the dual of the control space. To study the state equation, which is an equation with measures as data, and the adjoint equation, which involves the divergence of $L^{p}$-vector fields, we first study equations without convection term, and we next use a fixed point method to deal with the complete equations.
\end{abstract}

Mathematics Subject Classification. 49K20, 49J20, 35K57.

Received May 31, 2000. Revised February 15, 2001.

\section{INTRODUCTION}

We are interested in the following optimal control problem:

Can we control the temperature distribution of a fluid in a three dimensional domain by heating sources localized on a network of wires?

More generally we are interested in heating sources (the control variables) concentrated on thin structures. For simplicity we consider the case of controls localized on a manifold $\gamma$ included in a $N$-dimensional bounded domain $\bar{\Omega}$, but the case of a finite union of manifolds (a network of wires) can be considered as well. This optimal control problem clearly refers to a system of equations where the temperature and the fluid velocity are coupled. Such problems have been studied in the case of distributed or boundary controls (see for examples the references in [19]). The case of controls localized on thin structures, which is interesting for technological applications, has not yet been studied in the literature. As it is shown in [20], a fundamental step to tackle the complete Boussinesq system with controls localized on thin structures, first consists in studying a problem in which the fluid velocity is known. If $y$ denotes the fluid temperature and $\vec{V}$ the fluid velocity, the temperature $y$

Keywords and phrases: Pointwise control, optimal control, convection-diffusion equation, control localized on manifolds.

1 Université Paul Sabatier, UMR CNRS MIP, UFR MIG, 31062 Toulouse Cedex 4, France; e-mail: nguyen@mip.ups-tlse.fr and raymond@mip.ups-tlse.fr 
is the solution to the following convection-diffusion equation:

$$
\frac{\partial y}{\partial t}+A y+\vec{V} \cdot \nabla y=\left.u \delta_{\gamma}\right|_{Q} \text { in } Q, \quad \frac{\partial y}{\partial n_{A}}=\left.u \delta_{\gamma}\right|_{\Sigma} \text { on } \Sigma, \quad y(0)=y_{0} \text { in } \Omega,
$$

where $\Omega$ is a bounded domain in $\mathbb{R}^{N}$ with a regular boundary $\left.\Gamma, N \geq 2, Q=\Omega \times\right] 0, T[, T>0$ is given fixed, $\Sigma=\Gamma \times] 0, T\left[, A\right.$ is a second order elliptic operator of the form $A y=-\sum_{i, j=1}^{N} D_{i}\left(a_{i j}(x) D_{j} y\right)+a_{0}(x) y, \gamma \subset \bar{\Omega}$ is a regular manifold of dimension $0 \leq D \leq N-2, \delta_{\gamma}$ denotes the Dirac distribution on $\gamma$, and $u$ is a function from $\gamma \times] 0, T[$ with values in $\mathbb{R}$.

Convection-diffusion equations are often refered to flow related models, and a computational approach in the case of Neumann boundary control is carried out in [5]. In $[4,8]$, the case of pointwise controls (which is a particular type of thin structure) is considered for the one-dimensional Burgers' equation.

In order to next study the complete Boussinesq system we must suppose that $\vec{V}$ is not too regular. In control problems for convection-diffusion equation studied in the literature, it is often supposed that $\vec{V}$ is bounded $[5,11,12]$. However, it is not reasonable to suppose that the solution $(y, \vec{V})$ to the Boussinesq system is such that $\vec{V}$ belongs to $L^{\infty}\left(0, T ;\left(L^{\infty}(\Omega)\right)^{N}\right)$. Here we only suppose that $\vec{V}$ belongs to $L^{\tilde{m}}\left(0, T ;\left(L^{m}(\Omega)\right)^{N}\right)$, for some $\tilde{m}>2, m>2$ satisfying $\frac{1}{\tilde{m}}+\frac{N}{2 m} \leq \frac{1}{2}$ (for $N=2$ or $N=3$, the limit cases $\frac{1}{\tilde{m}}+\frac{N}{2 m}=\frac{1}{2}$, with $\tilde{m}=m=4$ if $N=2$, and $\tilde{m}=8$ and $m=4$ if $N=3$, correspond to the regularity of strong solutions of the Navier-Stokes equations).

These assumptions are sufficient to next study problems where the heat equation is coupled with the NavierStokes equations in the two following cases [20]:

- the Boussinesq system linearized at $(z, \vec{U})$, where $(z, \vec{U})$ has the same regularity as strong solutions of the Boussinesq system;

- the two-dimensional nonlinear Boussinesq system.

As it is shown in [13], even in the case when $\vec{V} \equiv 0$, studying equation (1) is not completely obvious. (In [13], the domain is supposed to be a 3-dimensional cylindrical domain, and taking advantage of the particular form of the domain, the equation is split into a 2-dimensional elliptic equation with measures as data, and a heat equation with regular source terms.)

Here we shall use new regularity results for parabolic equations with measures as data obtained in [21], where we have studied optimal control problems with controls localized on thin structures for semilinear parabolic equations.

In the present paper, we first study the control problem

$$
\inf \left\{I(y, u) \mid(y, u) \in L^{\kappa}\left(0, T ; W^{1, \kappa}(\Omega)\right) \times K_{U},(y, u) \text { satisfies }(1)\right\},
$$

where

$\left(C_{Q}, C_{\Omega}, C_{\gamma}\right.$ are nonnegative constants. $)$

$$
I(y, u)=C_{Q} \int_{Q}\left|\nabla y-V_{d}\right|^{\kappa} \mathrm{d} x \mathrm{~d} t+C_{\Omega} \int_{\Omega}\left|y(T)-y_{d}\right|^{\theta} \mathrm{d} x+C_{\gamma} \int_{0}^{T}\left(\int_{\gamma}|u|^{\sigma} \mathrm{d} \zeta\right)^{\frac{q}{\sigma}} \mathrm{d} t .
$$

Next, we consider the case when $\gamma$ is a point $x_{0}$ (that is $\delta_{\gamma}=\delta_{x_{0}}$. A finite union of points can be considered as well). In this case we are interested in characterizing the best location $x_{0}$ which minimizes the distance to an observed profile of temperature. The problem is formulated as follows:

$$
\inf \left\{J\left(y_{u, x_{0}}, u, x_{0}\right) \mid\left(y_{u, x_{0}}, u, x_{0}\right) \in L^{1}\left(0, T ; W^{1,1}(\Omega)\right) \times K_{U} \times K_{\bar{\Omega}}, \quad\left(y, u, x_{0}\right) \text { satisfies (1) }\right\},
$$

where

$$
J\left(y_{u, x_{0}}, u\right)=\int_{\Omega}\left|y_{u, x_{0}}(T)-y_{d}\right|^{\theta} \mathrm{d} x, \quad \text { and } \quad K_{\bar{\Omega}} \subset \bar{\Omega} .
$$


This problem can be related to the identification of sources of pollution (see [16]).

Also mention that the techniques we have developed for parabolic equations with measures as data can be adapted to study the corresponding stationnary elliptic equations with measures as data. In both cases (elliptic and parabolic) these new results can be useful to tackle optimal control problems with point observations. In this case the Dirac measures are involved in the adjoint equations [9]. For a review on control problems with pointwise controls we refer to [17] (see also [3]).

Let us briefly present the difficulties encountered in studying $\left(P_{1}\right)$ and $\left(P_{2}\right)$. Equation $(1)$ is an equation with measures as data which may be studied by the transposition method [6]. However the regularity results in the literature $[6,18]$ are not sufficient to deal with the control problems $\left(P_{1}\right)$ and $\left(P_{2}\right)$.

To obtain optimal regularity results for a convection-diffusion equation of the form (1), or for the adjoint equation associated with $\left(P_{1}\right)$, throughout the paper the idea consists in studying equations firstly when $\vec{V} \equiv 0$, and next, by using a fixed point method, extending theses results to the general case. The fixed point method is developed in details in the proof of Proposition 2.7, and is next used for different propositions in the paper. We prove regularity results for the state equation in Section 2. The control problem $\left(P_{1}\right)$ is studied in Section 3. The main difficulty to obtain optimality conditions for $\left(P_{1}\right)$ is to prove that the trace of the adjoint state on $\gamma \times] 0, T$ [ belongs to $L^{q^{\prime}}\left(0, T ; L^{\sigma^{\prime}}(\gamma)\right)$. The adjoint equation for $\left(P_{1}\right)$ is of the form

$$
-\frac{\partial p}{\partial t}+A p-\vec{V} \cdot \nabla p=-\operatorname{div} \vec{h} \text { in } Q, \quad \frac{\partial p}{\partial n_{A}}=\vec{h} \cdot \vec{n} \text { on } \Sigma, \quad p(T)=p_{T} \text { in } \Omega .
$$

Still using the fixed point method described above, we study the minimal regularity required on $\vec{h}$ and $p_{T}$ to have $\left.p\right|_{\gamma \times] 0, T[} \in L^{q^{\prime}}\left(0, T ; L^{\sigma^{\prime}}(\gamma)\right)$ (Ths. 3.2,3.3). Since in the adjoint equation $\vec{h}$ is equal to $\kappa C_{Q} \mid \nabla y_{u}-$ $\left.V_{d}\right|^{\kappa-2}\left(\nabla y_{u}-V_{d}\right)$, where $y_{u}$ is the solution to (1) corresponding to the optimal control $u$, and $p_{T}$ is equal to $\theta C_{\Omega}\left|y_{u}(T)-y_{d}\right|^{\theta-2}\left(y_{u}(T)-y_{d}\right)$, the conditions on $\vec{h}$ and $p_{T}$ to have $\left.p\right|_{\gamma \times] 0, T[} \in L^{q^{\prime}}\left(0, T ; L^{\sigma^{\prime}}(\gamma)\right)$, are satisfied under additional conditions on $\kappa$ and $\theta$ (these conditions are stated in assumptions (A9, A10)). Optimality conditions for $\left(P_{1}\right)$ are obtained in Theorem 3.5.

The control problem $\left(P_{2}\right)$ is studied in Section 4. To obtain optimality conditions for $\left(P_{2}\right)$, we prove that the adjoint state belongs to $L^{1}\left(0, T ; C^{1, \nu}(\bar{\Omega})\right)$ (Th. 4.2). Next, we are able to characterize the optimal location of a pointwise control (Th. 4.3).

Numerical experiments for the computation of optimal solutions $u$ for $\left(P_{1}\right)$, and optimal pairs $\left(u, x_{0}\right)$ for $\left(P_{2}\right)$ are reported in [20] (Chap. 6).

\section{State equation}

\subsection{Notation and assumptions}

We make the following assumptions on the data.

(A1) The elliptic operator $A$ is defined by $A y=-\sum_{i, j=1}^{N} D_{i}\left(a_{i j}(x) D_{j} y\right)+a_{0}(x) y$. The coefficient $a_{0}$ is positive and belongs to $C(\bar{\Omega})$, the coefficients $a_{i j}$ belong to $C^{1, \nu}(\bar{\Omega})$ with $0<\nu \leq 1, a_{i j}=a_{j i}$, and they satisfy

$$
\sum_{i, j=1}^{N} a_{i j}(x) \xi_{i} \xi_{j} \geq m_{0}|\xi|^{2} \quad \text { for every } \xi \in R^{N} \text { and every } x \in \bar{\Omega}, \quad \text { with } m_{0}>0
$$

(A2) $\Gamma$ is of class $C^{\infty}$, and $\gamma$ is a submanifold in $\bar{\Omega}$ of dimension $D \leq N-2$, of class $C^{\bar{k}}$ with $\bar{k}=$ $\max \left(2,\left[\frac{N-D}{\sigma^{\prime}}\right]+1\right)$. 
(A3) $\vec{V}$ belongs to $L^{\tilde{m}}\left(0, T ;\left(L^{m}(\Omega)\right)^{N}\right)$ and satisfies

$$
\operatorname{div} \vec{V}=0 \text { in } Q, \quad \vec{V} \cdot \vec{n}=0 \text { on } \Sigma, \quad 2<\tilde{m}<\infty, \quad 2 \leq N<m<\infty, \quad \frac{1}{\tilde{m}}+\frac{N}{2 m} \leq \frac{1}{2},
$$

where "div" denotes the divergence operator with respect to $x \in \Omega$.

(A4) $u$ belongs to $L^{q}\left(0, T ; L^{\sigma}(\gamma)\right)$ with $q \geq 2, \quad \sigma \geq \frac{N}{N-1}$.

(A5) $y_{0}$ belongs to $L^{\rho}(\Omega)$ with $\rho=\frac{N}{N-\frac{D}{\sigma^{\prime}}-\frac{2}{q^{\prime}}}$.

(A6) $K_{U}$ is a closed convex subset of $L^{q}\left(0, T ; L^{\sigma}(\gamma)\right)$. Either $K_{U}$ is bounded in $L^{q}\left(0, T ; L^{\sigma}(\gamma)\right)$, or $C_{\gamma}>0$.

(A7) The function $V_{d}$ belongs to $L^{\kappa}\left(0, T ;\left(L^{\kappa}(\Omega)\right)^{N}\right)$, the function $y_{d}$ belongs to $L^{\theta}(\Omega)$, with $\kappa>1, \theta>1$.

Remark. For simplicity we have supposed that $\Gamma$ is of class $C^{\infty}$, but the results of the paper can be extended to less regular domains by using the techniques of [7] (Prop. 5).

Throughout the paper, we denote by $T_{\gamma}$ (respectively $T_{\gamma \times] 0, T[}, T_{\Sigma}$ ) the trace mapping on $\gamma$ (respectively on $\gamma \times] 0, T[$, on $\Sigma)$. We denote by $C, C_{i}, K, K_{i}$ for $i \in \mathbb{N}$, various constants depending on known quantities. The same letter may be used for different constants.

In [21] we have studied equation (1) in the case when $\vec{V} \equiv 0$. By the transposition method, we have proven the following regularity results.

Proposition 2.1. [21] (Prop. 2.3) Suppose that $\vec{V} \equiv 0$ and $y_{0} \equiv 0$. Equation (1) admits a unique solution $y_{u}$ in $L^{1}\left(0, T ; W^{1,1}(\Omega)\right)$. The mapping $u \mapsto y_{u}$ is continuous from $L^{q}\left(0, T ; L^{\sigma}(\gamma)\right)$ into $L^{\delta_{1}}\left(0, T ; W^{1, d_{1}}(\Omega)\right)$ for every $\left(\delta_{1}, d_{1}\right)$ satisfying:

$$
\begin{aligned}
& q \leq \delta_{1}, \quad \sigma \leq d_{1}<\frac{N}{N-\frac{D}{\sigma^{\prime}}-1}, \quad \frac{N-D}{2}+\frac{D}{2 \sigma}+\frac{1}{q}<\frac{1}{\delta_{1}}+\frac{N}{2 d_{1}}+\frac{1}{2}, \text { if } \sigma<\frac{N-D}{N-D-1}, \\
& q \leq \delta_{1}, \quad 1<d_{1}<\frac{N-D}{N-D-1}, \quad \frac{N-D}{2}+\frac{1}{q}<\frac{1}{\delta_{1}}+\frac{N-D}{2 d_{1}}+\frac{1}{2}, \quad \text { if } \sigma \geq \frac{N-D}{N-D-1} .
\end{aligned}
$$

The mapping that associates $y_{u}$ with $u$ is continuous from $L^{q}\left(0, T ; L^{\sigma}(\gamma)\right)$ into $L^{\infty}\left(0, T ; L^{r}(\Omega)\right)$ for every $1 \leq r<$

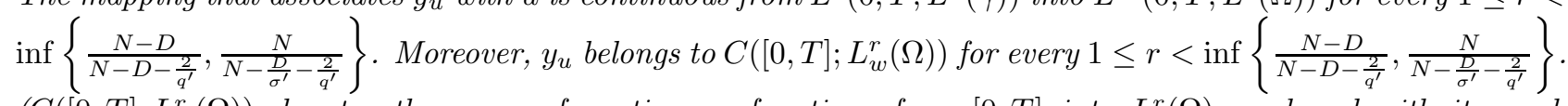
$\left(C\left([0, T] ; L_{w}^{r}(\Omega)\right)\right.$ denotes the space of continuous functions from $[0, T]$ into $L^{r}(\Omega)$, endowed with its weak topology.)

Remark. The conditions expressed in (2) can be written in the following shorter form

$$
q \leq \delta_{1}, \quad \sigma \leq d_{1}, \quad \frac{N-D}{2}+\frac{D}{2 \sigma}+\frac{1}{q}<\frac{1}{\delta_{1}}+\frac{N}{2 d_{1}}+\frac{1}{2} .
$$

Indeed if $d_{1} \geq \sigma$, taking $\delta_{1}=q$ in (3), we obtain:

$$
\frac{N-D}{2}+\frac{D}{2 d_{1}}<\frac{N-D}{2}+\frac{D}{2 \sigma}<\frac{N}{2 d_{1}}+\frac{1}{2}
$$

Therefore we have $\sigma \leq d_{1}<\frac{N-D}{N-D-1}$. This means that (3) cannot be used if $\sigma \geq \frac{N-D}{N-D-1}$. Now, if $u \in$ $L^{q}\left(0, T ; L^{\sigma}(\gamma)\right)$ with $\sigma \geq \frac{N-D}{N-D-1}$, then $u \in L^{q}\left(0, T ; L^{\hat{\sigma}}(\gamma)\right)$ for any $\hat{\sigma}<\frac{N-D}{N-D-1}$. Therefore $y$ belongs to $L^{\delta_{1}}\left(0, T ; W^{1, d_{1}}(\Omega)\right)$ for every $\left(\delta_{1}, d_{1}\right)$ satisfying:

$$
q \leq \delta_{1}, \quad \hat{\sigma}=d_{1}<\frac{N-D}{N-D-1} \text { and } \frac{N-D}{2}+\frac{D}{2 \hat{\sigma}}+\frac{1}{q}<\frac{1}{\delta_{1}}+\frac{N}{2 d_{1}}+\frac{1}{2},
$$


that is

$$
\frac{N-D}{2}+\frac{1}{q}<\frac{1}{\delta_{1}}+\frac{N-D}{2 d_{1}}+\frac{1}{2}
$$

which is nothing else than the second condition in (2). Finally observe that the condition $d_{1}<\frac{N}{N-\frac{D}{\sigma^{\prime}}-1}$ follows from (3) by taking $\delta_{1}=q$. Even if (2) and (3) are equivalent, using (2) avoids forgetting the condition $d_{1}<\frac{N-D}{N-D-1}$.

Proposition 2.2. [21] (Prop. 2.5) Suppose that $\vec{V} \equiv 0$ and $u \equiv 0$. Let $y_{0}$ belong to $L^{\rho}(\Omega)$ with $\rho=\frac{N}{N-\frac{D}{\sigma^{\prime}}-\frac{2}{q^{\prime}}}$, and let $y$ be the solution to equation (1). The mapping $y_{0} \mapsto y$ is continuous from $L^{\rho}(\Omega)$ into $L^{\infty}\left(0, T ; L^{\rho}(\Omega)\right)^{q^{q}} \cap$ $L^{\delta_{2}}\left(0, T ; W^{1, d_{2}}(\Omega)\right)$, for every $\left(\delta_{2}, d_{2}\right)$ satisfying

$$
1<\delta_{2}<2, \quad \rho \leq d_{2}<\frac{N \rho}{N-\rho}, \quad 1+\frac{N}{2 \rho}<\frac{1}{\delta_{2}}+\frac{N}{2 d_{2}}+\frac{1}{2} .
$$

In this section we want to extend these results to the case when $\vec{V}$ satisfies (A3) (Props. 2.7 and 2.8). Due to the weak regularity of $\vec{V}$, we cannot use the transposition method. We obtain existence and regularity results for equation (1) by using a fixed point method. For this, we need some preliminary estimates that are stated below.

\subsection{Preliminary estimates}

First recall some results for analytic semigroups. We denote by $\tilde{A}$ the operator defined by

$$
\mathcal{D}(\tilde{A})=\left\{y \in C^{2}(\bar{\Omega}) \mid \frac{\partial y}{\partial n_{A}}=0 \text { on } \Gamma\right\}, \quad \tilde{A} y=A y .
$$

For $1 \leq \ell<\infty$, we denote by $A_{\ell}$ the closure of $\tilde{A}$ in $L^{\ell}(\Omega)$. The operator $-A_{\ell}$ is the generator of a strongly continuous analytic semigroup $S_{\ell}(t)_{t \geq 0}$ in $L^{\ell}(\Omega)[2]$. For $1<\ell<\infty$ the domain of $A_{\ell}$ is $\mathcal{D}\left(A_{\ell}\right)=$ $\left\{y \in W^{2, \ell}(\Omega) \mid \frac{\partial y}{\partial n_{A}}=0\right.$ on $\left.\Gamma\right\}$. For any $1 \leq \ell<\infty, 0$ belongs to the resolvent of $-A_{\ell}$ and there exists $\delta>0$ such that $\operatorname{Re} \sigma\left(A_{\ell}\right) \geq \delta$ (it is a consequence of (A1) and of the fact that $\sigma\left(A_{\ell}\right)$ is independent of $\ell$ ). Therefore, for $\alpha>0$, there exists a constant $K=K(\ell, \alpha)$ such that

$$
\left\|A_{\ell}^{\alpha} S_{\ell}(t) \varphi\right\|_{L^{\ell}(\Omega)} \leq K t^{-\alpha}\|\varphi\|_{L^{\ell}(\Omega)}
$$

for every $t>0$ and every $\varphi \in L^{\ell}(\Omega)$ (see [14,22], $A_{\ell}^{\alpha}$ is the $\alpha$-power of $A_{\ell}$ ). Thanks to this result the following lemma can be established.

Lemma 2.1. [2,24] For every $1 \leq \ell \leq \lambda \leq \infty$ with $\ell<\infty$, there exists a constant $K_{1}=K_{1}(\lambda, \ell)$ such that

$$
\left\|S_{\ell}(t) \varphi\right\|_{L^{\lambda}(\Omega)} \leq K_{1} t^{-\frac{N}{2}\left(\frac{1}{\ell}-\frac{1}{\lambda}\right)}\|\varphi\|_{L^{\ell}(\Omega)}
$$

for every $\varphi \in L^{\ell}(\Omega)$ and every $t>0$. For every $1 \leq \ell \leq \lambda \leq \infty$ with $\ell<\infty$, and every $\alpha>0$, there exists a constant $K_{2}=K_{2}(\lambda, \ell, \alpha)$ such that

$$
\left\|A_{\ell}^{\alpha} S_{\ell}(t) \varphi\right\|_{L^{\lambda}(\Omega)} \leq K_{2} t^{-\frac{N}{2}\left(\frac{1}{\ell}-\frac{1}{\lambda}\right)-\alpha}\|\varphi\|_{L^{\ell}(\Omega)}
$$

for every $\varphi \in L^{\ell}(\Omega)$ and every $t>0$.

Proposition 2.3. [1] (Th. 7.58) If assumption (A2) is satisfied, then $T_{\gamma}$ is a continuous linear operator from $W^{r, p}(\Omega)$ into $L^{q}(\gamma)$ for all $(r, p, q)$ such that $0 \leq r \leq \bar{k}, 0<N-r p<D, p \leq q<\frac{D p}{N-r p}$. 
Remark. Theorem 7.58 in [1] is stated with $\Omega$ and $\gamma$ replaced by $\mathbb{R}^{N}$ and $\mathbb{R}^{D}$, but as it is noticed in [1], just before Theorem 7.58, the statement is also true for domains by using coverings, partitions of unity, and diffeomorphisms of class $C^{\bar{k}}$.

Proposition 2.4. [21] (Prop. 2.1) Let $\phi$ be in $\mathcal{D}(\Omega)$, and $w$ be the solution of the Cauchy problem:

$$
\frac{\partial w}{\partial t}+A w=0 \text { in } Q, \quad \frac{\partial w}{\partial n_{A}}=0 \text { on } \Sigma, \quad w(0)=A_{d^{\prime}}^{\alpha} \phi \text { in } \Omega
$$

where $0 \leq \alpha \leq 1$. The mapping that associates $w$ with $\phi$ is continuous from $L^{d^{\prime}}(\Omega)$ into $L^{i}\left(0, T ; W^{r, j}(\Omega)\right)$ for all $(\alpha, q, j, i, r, d)$ satisfying:

$$
2>r \geq 0, \quad i \geq 1, \quad j \geq d^{\prime}, \quad \alpha+\frac{r}{2}+\frac{N}{2 d^{\prime}}<\frac{1}{i}+\frac{N}{2 j} .
$$

Proposition 2.5. Let $\vec{f}$ be in $(\mathcal{D}(Q))^{N}$, and $z$ be the solution of the equation:

$$
\frac{\partial z}{\partial t}+A z=\operatorname{div} \vec{f} \text { in } Q, \quad \frac{\partial z}{\partial n_{A}}=0 \text { on } \Sigma, \quad z(0)=0 \text { in } \Omega .
$$

The mapping that associates $z$ with $\vec{f}$ is continuous from $L^{\tilde{\eta}}\left(0, T ;\left(L^{\eta}(\Omega)\right)^{N}\right)$ into $L^{\tilde{\eta}}\left(0, T ; W^{1, \eta}(\Omega)\right)$. It is also continuous from $L^{\tilde{\eta}}\left(0, T ;\left(L^{\eta}(\Omega)\right)^{N}\right)$ into $L^{\delta}\left(0, T ; W^{r, d}(\Omega)\right)$ for all $(r, \tilde{\eta}, \eta, \delta, d)$ satisfying:

$$
0 \leq r<1,1 \leq \tilde{\eta} \leq \delta, 1<\eta \leq d, \frac{r}{2}+\frac{1}{\tilde{\eta}}+\frac{N}{2 \eta}<\frac{1}{\delta}+\frac{N}{2 d}+\frac{1}{2}
$$

The mapping that associates $z$ with $\vec{f}$ is continuous from $L^{\tilde{\eta}}\left(0, T ;\left(L^{\eta}(\Omega)\right)^{N}\right)$ into $L^{\delta}\left(0, T ; L^{d}(\Omega)\right)$ for all $(\tilde{\eta}, \eta, \delta, d)$ satisfying:

$$
1<\tilde{\eta}<\delta, 1<\eta \leq d, \frac{1}{\tilde{\eta}}+\frac{N}{2 \eta} \leq \frac{1}{\delta}+\frac{N}{2 d}+\frac{1}{2}
$$

Remark. Since $\vec{f}$ belongs to $(\mathcal{D}(Q))^{N}$, equation (9) is defined in a classical sense.

Proof. The first continuity results are already proved in [21] and [27]. Here we only prove the second one. Let $w$ be the solution of the Cauchy problem (7) when $\alpha=0$, then:

$$
\begin{aligned}
\int_{\Omega} z(x, t) \phi(x) \mathrm{d} x & =\int_{0}^{t}\left\{\frac{d}{d \tau} \int_{\Omega} w(x, t-\tau) z(x, \tau) \mathrm{d} x\right\} \mathrm{d} \tau \\
& =\int_{0}^{t} \int_{\Omega}\left\{-\frac{\partial w}{\partial t}(x, t-\tau) z(x, \tau)+w(x, t-\tau) \frac{\partial z}{\partial t}(x, \tau)\right\} \mathrm{d} x \mathrm{~d} \tau \\
& =\int_{0}^{t} \int_{\Omega}\{A w(x, t-\tau) z(x, \tau)-w(x, t-\tau) A z(x, \tau)+\operatorname{div} \vec{f}(x, \tau) w(x, t-\tau)\} \mathrm{d} x \mathrm{~d} \tau \\
& =-\int_{0}^{t} \int_{\Omega} \nabla w(x, t-\tau) \cdot \vec{f}(x, \tau) \mathrm{d} x \mathrm{~d} \tau .
\end{aligned}
$$


Using (6) in Lemma 2.1 with $1 \leq d^{\prime} \leq \eta^{\prime} \leq \infty, d^{\prime}<\infty$, we have:

$$
\begin{aligned}
\|z(t)\|_{L^{d}(\Omega)} & =\sup \left\{\left|\int_{\Omega} z(x, t) \phi(x) \mathrm{d} x\right|,\|\phi\|_{L^{d^{\prime}}(\Omega)}=1\right\} \\
& =\sup \left\{\left|\int_{0}^{t} \int_{\Omega} \nabla w(x, t-\tau) \cdot \vec{f}(x, \tau) \mathrm{d} x \mathrm{~d} \tau\right|,\|\phi\|_{L^{d^{\prime}}(\Omega)}=1\right\} \\
& \leq K \int_{0}^{t}(t-\tau)^{-\frac{1}{2}-\frac{N}{2}\left(\frac{1}{\eta}-\frac{1}{d}\right)}\|\vec{f}(\tau)\|_{\left(L^{\eta}(\Omega)\right)^{N}} \mathrm{~d} \tau .
\end{aligned}
$$

The mapping $\tau \mapsto\|\vec{f}(\tau)\|_{\left(L^{\eta}(\Omega)\right)^{N}}$ belongs to $L^{\tilde{\eta}}(0, T)$. We denote by $L_{*}^{i}(0, T)$ the weak- $L^{i}(0, T)$ space defined as follows ([23], p. 30):

$$
L_{*}^{i}(0, T):=\left\{g:(0, T) \mapsto R \mid g \text { is measurable, and } \sup _{\xi>0}\left(\xi^{i} \mathcal{L}^{1}\{t|| g(t) \mid>\xi\}\right)<\infty\right\},
$$

where $\mathcal{L}^{1}$ denotes the Lebesgue measure on $(0, T)$. Then the mapping $t \mapsto t^{-\frac{1}{2}-\frac{N}{2}\left(\frac{1}{\eta}-\frac{1}{d}\right)}$ belongs to $L_{*}^{i}(0, T)$ with $i>1$ defined by $\frac{1}{2}+\frac{N}{2}\left(\frac{1}{\eta}-\frac{1}{d}\right)=\frac{1}{i}$. Due to (11), $1+\frac{1}{\delta} \geq \frac{1}{i}+\frac{1}{\tilde{\eta}}$. From the generalized Young inequality, it follows that the mapping $t \mapsto \int_{0}^{t}(t-\tau)^{-\frac{1}{2}-\frac{N}{2}\left(\frac{1}{\eta}-\frac{1}{d}\right)}\|\vec{f}(\tau)\|_{\left(L^{\eta}(\Omega)\right)^{N}} \mathrm{~d} \tau$ belongs to $L^{\delta}(0, T)$, and the proof is complete.

Remark. Since $(\mathcal{D}(Q))^{N}$ is dense in $L^{\tilde{\eta}}\left(0, T ;\left(L^{\eta}(\Omega)\right)^{N}\right)$, the regularity result of Proposition 2.5 is also true for the solution $z$ to the variational equation

$$
-\int_{Q} z \frac{\partial \phi}{\partial t} \mathrm{~d} x \mathrm{~d} t+\int_{Q} \sum_{i, j=1}^{N} a_{i j} D_{j} z D_{i} \phi \mathrm{d} x \mathrm{~d} t+\int_{Q} a_{0} z \phi \mathrm{d} x \mathrm{~d} t \mathrm{~d} x \mathrm{~d} t=-\int_{Q} \vec{f} \cdot \nabla \phi \mathrm{d} x \mathrm{~d} t,
$$

for all $\phi \in C^{1}(\bar{Q})$ such that $\phi(T)=0$ on $\bar{\Omega}$, where $\vec{f} \in L^{\tilde{\eta}}\left(0, T ;\left(L^{\eta}(\Omega)\right)^{N}\right)$.

Proposition 2.6. Let $f$ be in $\mathcal{D}(Q)$, and $z$ be the solution of the equation:

$$
\frac{\partial z}{\partial t}+A z=f \text { in } Q, \quad \frac{\partial z}{\partial n_{A}}=0 \text { on } \Sigma, \quad z(0)=0 \text { in } \Omega .
$$

The mapping that associates $z$ with $f$ is continuous from $L^{\tilde{\eta}}\left(0, T ; L^{\eta}(\Omega)\right)$ into $L^{\delta}\left(0, T ; W^{r, d}(\Omega)\right)$ for all $(r, \tilde{\eta}, \eta, \delta, d)$ satisfying:

$$
0 \leq r<2,1 \leq \tilde{\eta} \leq \delta, 1<\eta \leq d, \frac{r}{2}+\frac{1}{\tilde{\eta}}+\frac{N}{2 \eta}<\frac{1}{\delta}+\frac{N}{2 d}+1
$$

The mapping that associates $z$ with $f$ is continuous from $L^{\tilde{\eta}}\left(0, T ; L^{\eta}(\Omega)\right)$ into $L^{\delta}\left(0, T ; W^{k, d}(\Omega)\right)$ for all $(k, \tilde{\eta}, \eta, \delta, d)$ satisfying:

$$
k=0 \text { or } k=1,1<\tilde{\eta}<\delta, 1<\eta \leq d, \frac{k}{2}+\frac{1}{\tilde{\eta}}+\frac{N}{2 \eta} \leq \frac{1}{\delta}+\frac{N}{2 d}+1 .
$$

Proof. The first continuity result is already proved in [21]. Here we only prove the second one. Let us set $\alpha=\frac{k}{2}$. We have $D\left(A_{d}^{0}\right)=L^{d}(\Omega)$ and $D\left(A_{d}^{\frac{1}{2}}\right)=W^{1, d}(\Omega)$. Let $w$ be the solution of the Cauchy problem (7), then:

$$
\int_{\Omega} A_{d}^{\alpha} z(x, t) \phi(x) \mathrm{d} x=\int_{0}^{t} \int_{\Omega} A_{d^{\prime}}^{\alpha} S_{d^{\prime}}(t-\tau) \phi(x) f(x, \tau) \mathrm{d} x \mathrm{~d} \tau .
$$


Using (6) in Lemma 2.1 with $1 \leq d^{\prime} \leq \eta^{\prime} \leq \infty, d^{\prime}<\infty$, we have:

$$
\begin{aligned}
\|z(t)\|_{W^{k, d}(\Omega)} & \leq C\left\|A_{d}^{\alpha} z(t)\right\|_{L^{d}(\Omega)} \leq C \sup \left\{\left|\int_{\Omega} A_{d}^{\alpha} z(x, t) \phi(x) \mathrm{d} x\right|,\|\phi\|_{L^{d^{\prime}}(\Omega)}=1\right\} \\
& =C \sup \left\{\left|\int_{0}^{t} \int_{\Omega} A_{d^{\prime}}^{\alpha} S_{d^{\prime}}(t-\tau) \phi f(\tau) \mathrm{d} x \mathrm{~d} \tau\right|,\|\phi\|_{L^{d^{\prime}}(\Omega)}=1\right\} \\
& \leq C \int_{0}^{t}(t-\tau)^{-\alpha-\frac{N}{2}\left(\frac{1}{\eta}-\frac{1}{d}\right)}\|f(\tau)\|_{L^{\eta}(\Omega)} \mathrm{d} \tau .
\end{aligned}
$$

The mapping $\tau \mapsto\|f(\tau)\|_{L^{\eta}(\Omega)}$ belongs to $L^{\tilde{\eta}}(0, T)$. The mapping $t \mapsto t^{-\alpha-\frac{N}{2}\left(\frac{1}{\eta}-\frac{1}{d}\right)}$ belongs to $L_{*}^{i}(0, T)$ with $i>1$ defined by $\alpha+\frac{N}{2}\left(\frac{1}{\eta}-\frac{1}{d}\right)=\frac{1}{i}$. Due to (14), $1+\frac{1}{\delta} \geq \frac{1}{i}+\frac{1}{\tilde{\eta}}$. From the generalized Young inequality, it follows that the mapping $t \mapsto \int_{0}^{t}(t-\tau)^{-\alpha-\frac{N}{2}\left(\frac{1}{\eta}-\frac{1}{d}\right)}\|f(\tau)\|_{L^{\eta}(\Omega)} \mathrm{d} \tau$ belongs to $L^{\delta}(0, T)$.

\subsection{State equation}

In this section, we prove regularity results for equation (1). We shall say that a function $y \in L^{\delta}\left(0, T ; W^{1, d}(\Omega)\right)$ is a weak solution to equation (1) if and only if $\delta \geq \tilde{m}^{\prime}, d \geq m^{\prime}$ and

$$
\begin{aligned}
& -\int_{Q} y \frac{\partial \phi}{\partial t} \mathrm{~d} x \mathrm{~d} t+\int_{Q} \sum_{i, j=1}^{N} a_{i j}(x) D_{j} y D_{i} \phi \mathrm{d} x \mathrm{~d} t+\int_{Q} a_{0} y \phi \mathrm{d} x \mathrm{~d} t+\int_{Q} \vec{V} \cdot \nabla y \phi \mathrm{d} x \mathrm{~d} t \\
& =\int_{0}^{T} \int_{\gamma} u \phi \mathrm{d} \zeta \mathrm{d} t+\int_{\Omega} \phi(0) y_{0} \mathrm{~d} x \quad \text { for all } \phi \in C^{1}(\bar{Q}) \text { such that } \phi(T)=0 \text { on } \bar{\Omega} .
\end{aligned}
$$

To simplify the writing, throughout the sequel, we suppose that $\gamma$ is included in $\Gamma$, but the results are true for $\gamma \subset \bar{\Omega}$.

Proposition 2.7. We consider the equation

$$
\frac{\partial y}{\partial t}+A y+\vec{V} \cdot \nabla y=0 \text { in } Q, \quad \frac{\partial y}{\partial n_{A}}=u \delta_{\gamma} \text { on } \Sigma, \quad y(0)=0 \text { in } \Omega .
$$

Equation (16) admits a unique solution y in $L^{\hat{\delta}}\left(0, T ; W^{1, \hat{d}}(\Omega)\right)$ for all $(\hat{\delta}, \hat{d})$ obeying $\tilde{m}^{\prime}<\hat{\delta} \leq q, m^{\prime}<\hat{d}<$ $\inf \left\{\frac{N}{N-\frac{D}{\sigma^{\prime}}-1}, \frac{N-D}{N-D-1}\right\}$. Moreover, the mapping that associates y with $u$ is continuous from $L^{q}\left(0, T ; L^{\sigma}(\gamma)\right)$ into $L^{\delta}\left(0, T ; W^{1, d}(\Omega)\right)$ for every $(\delta, d)$ satisfying:

$$
\begin{array}{cl}
q \leq \delta, \quad \sigma \leq d<\frac{N}{N-\frac{D}{\sigma^{\prime}}-1}, \quad \frac{N-D}{2}+\frac{D}{2 \sigma}+\frac{1}{q}<\frac{1}{\delta}+\frac{N}{2 d}+\frac{1}{2}, & \text { if } \sigma<\frac{N-D}{N-D-1}, \\
q \leq \delta, \quad m^{\prime}<d<\frac{N-D}{N-D-1}, \quad \frac{N-D}{2}+\frac{1}{q}<\frac{1}{\delta}+\frac{N-D}{2 d}+\frac{1}{2}, & \text { if } \sigma \geq \frac{N-D}{N-D-1} .
\end{array}
$$

The mapping that associates $y$ with $u$ is continuous from $L^{q}\left(0, T ; L^{\sigma}(\gamma)\right)$ into $L^{\infty}\left(0, T ; L^{r}(\Omega)\right)$ for every $1 \leq r<$ $\inf \left\{\frac{N}{N-\frac{D}{N-D}-\frac{2}{q^{r}}}, \frac{N}{N-\frac{D}{\sigma^{r}}-\frac{2}{q^{\prime}}}\right\}$. Moreover, $y$ belongs to $C\left([0, T] ; L_{w}^{r}(\Omega)\right)$ for every $1 \leq r<$ $\inf \left\{\frac{N}{N-\frac{D}{N-D}-\frac{2}{q^{\prime}}}, \frac{N}{N-\frac{D}{\sigma^{\prime}}-\frac{2}{q^{\prime}}}\right\}$. 
Proof. 1 - Existence of a local solution. Let us set $\left.Q_{\bar{t}}:=\Omega \times\right] 0, \bar{t}\left[, \quad \Sigma_{\bar{t}}:=\Gamma \times\right] 0, \bar{t}[$. Let $(\delta, d)$ be a pair obeying (17). By a fixed point method, we prove that the equation

$$
\frac{\partial y}{\partial t}+A y+\vec{V} \cdot \nabla y=0 \text { in } Q_{\bar{t}}, \quad \frac{\partial y}{\partial n_{A}}=u \delta_{\gamma} \text { on } \Sigma_{\bar{t}}, \quad y(0)=0 \text { in } \Omega,
$$

admits a solution for $\bar{t}>0$ small enough. Let $\xi$ belong to $L^{\delta}\left(0, \bar{t} ; W^{1, d}(\Omega)\right)$, and $y_{\xi}$ be the solution to the equation:

$$
\frac{\partial y_{\xi}}{\partial t}+A y_{\xi}=-\vec{V} \cdot \nabla \xi \text { in } Q_{\bar{t}}, \quad \frac{\partial y_{\xi}}{\partial n_{A}}=u \delta_{\gamma} \text { on } \Sigma_{\bar{t}}, \quad y_{\xi}(0)=0 \text { in } \Omega
$$

Then $y_{\xi}=\hat{y}+\tilde{y}$, where $\hat{y}$ and $\tilde{y}$ are the solutions to the equations:

$$
\begin{gathered}
\frac{\partial \hat{y}}{\partial t}+A \hat{y}=0 \text { in } Q_{\bar{t}}, \quad \frac{\partial \hat{y}}{\partial n_{A}}=u \delta_{\gamma} \text { on } \Sigma_{\bar{t}}, \quad \hat{y}(0)=0 \text { in } \Omega, \\
\frac{\partial \tilde{y}}{\partial t}+A \tilde{y}=-\vec{V} \cdot \nabla \xi \text { in } Q_{\bar{t}}, \quad \frac{\partial \tilde{y}}{\partial n_{A}}=0 \text { on } \Sigma_{\bar{t}}, \quad \tilde{y}(0)=0 \text { in } \Omega .
\end{gathered}
$$

From Proposition 2.1, we know that $\hat{y} \in L^{\delta}\left(0, \bar{t} ; W^{1, d}(\Omega)\right)$. Since $\delta \geq q \geq 2>\tilde{m}^{\prime}$, and $d \geq \sigma \geq \frac{N}{N-1}>m^{\prime}$, then $\frac{\tilde{m} \delta}{\tilde{m}+\delta}>1, \frac{m d}{m+d}>1$, and $\vec{V} \cdot \nabla \xi \in L^{\frac{\tilde{m} \delta}{\dot{m}+\delta}}\left(0, \bar{t} ; L^{\frac{m d}{m+d}}(\Omega)\right)$. Due to Proposition 2.6, it follows that $\tilde{y} \in$ $L^{\delta}\left(0, \bar{t} ; W^{1, d}(\Omega)\right)$. Thus $y_{\xi}$ belongs to $L^{\delta}\left(0, \bar{t} ; W^{1, d}(\Omega)\right)$.

Let $\xi_{1}$ and $\xi_{2}$ belong to $L^{\delta}\left(0, \bar{t} ; W^{1, d}(\Omega)\right)$. Still with Proposition 2.6, we have

$$
\left\|y_{\xi_{1}}-y_{\xi_{2}}\right\|_{L^{\delta}\left(0, \bar{t} ; W^{1, d}(\Omega)\right)} \leq C_{1}\|\vec{V}\|_{L^{\tilde{m}}\left(0, \bar{t} L^{m}(\Omega)^{N}\right)}\left\|\xi_{1}-\xi_{2}\right\|_{L^{\delta}\left(0, \bar{t} ; W^{1, d}(\Omega)\right)},
$$

where $C_{1}$ can be chosen depending on $T$, but independent of $\bar{t}$. The mapping $t \mapsto C_{1}^{\tilde{m}} \int_{0}^{t}\|\vec{V}(x, \tau)\|_{L^{m}(\Omega)^{N}}^{\tilde{m}} \mathrm{~d} \tau$ is absolutely continuous, then there exists $\bar{t}>0$ such that $C_{1}\left(\int_{t}^{\min \{t+\bar{t}, T\}}\|\vec{V}(\cdot, \tau)\|_{L^{m}(\Omega)^{N}}^{\tilde{m}} \mathrm{~d} \tau\right)^{\frac{1}{\tilde{m}}} \leq C=\frac{1}{2}$ for all $t \in[0, T]$. Thus the mapping $\xi \mapsto y_{\xi}$ is a contraction in the Banach space $L^{\delta}\left(0, \bar{t} ; W^{1, d}(\Omega)\right)$.

2 - Estimate of the local solution. Consider the sequence $\left(\xi_{n}\right)_{n}$ defined by $\xi_{0}=0$ and $\xi_{n}=y_{\xi_{n-1}}$. Then $\left(\xi_{n}\right)_{n}$ converges to the unique solution $y$ of (18). From the definition of $y_{\xi_{0}}$, and due to Proposition 2.1, we deduce that

Moreover for all $n$ we have

$$
\left\|y_{\xi_{0}}\right\|_{L^{\delta}\left(0, \bar{t} ; W^{1, d}(\Omega)\right)} \leq K\|u\|_{L^{q}\left(0, \bar{t} ; L^{\sigma}(\gamma)\right)} .
$$

$$
\left\|y_{\xi_{n}}\right\|_{L^{\delta}\left(0, \bar{t} ; W^{1, d}(\Omega)\right)} \leq 2\left\|y_{\xi_{0}}\right\|_{L^{\delta}\left(0, \bar{t} ; W^{1, d}(\Omega)\right)} \leq K\|u\|_{L^{q}\left(0, T ; L^{\sigma}(\gamma)\right)} .
$$

By letting $n$ goes to $\infty$, we obtain:

$$
\|y\|_{L^{\delta}\left(0, \bar{t} ; W^{1, d}(\Omega)\right)} \leq K\|u\|_{L^{q}\left(0, T ; L^{\sigma}(\gamma)\right)} .
$$

3 - Existence of a global solution. We prove that a solution exists in $L^{\delta}\left(0, T ; W^{1, d}(\Omega)\right)$, by repeating the above process. Let $\hat{y}$ be the solution constructed on $(0, \bar{t})$ in Step 2 . Let $\left(\hat{\xi}_{1}, \hat{\xi}_{2}\right)$ belong to $L^{\delta}\left(\bar{t}, 2 \bar{t} ; W^{1, d}(\Omega)\right)$. Define $\left(\xi_{1}, \xi_{2}\right)$ belonging to $L^{\delta}\left(0,2 \bar{t} ; W^{1, d}(\Omega)\right)$ by $\xi_{1}=\xi_{2}=\hat{y}$ on $(0, \bar{t})$, and $\xi_{1}=\hat{\xi}_{1}, \xi_{2}=\hat{\xi}_{2}$ on $(\bar{t}, 2 \bar{t})$. We still denote by $y_{\xi_{i}}$ the solution to equation (19) on $(0,2 \bar{t})$ corresponding to $\xi_{i}$ for $i=1,2$. As in Step 2, we have:

$$
\left\|y_{\xi_{1}}-y_{\xi_{2}}\right\|_{L^{\delta}\left(0,2 \bar{t} ; W^{1, d}(\Omega)\right)}=\left\|y_{\xi_{1}}-y_{\xi_{2}}\right\|_{L^{\delta}\left(\bar{t}, 2 \bar{t} ; W^{1, d}(\Omega)\right)} \leq \frac{1}{2}\left\|\xi_{1}-\xi_{2}\right\|_{L^{\delta}\left(0,2 \bar{t} ; W^{1, d}(\Omega)\right)} .
$$


Thus the mapping $\xi \mapsto y_{\xi}$ admits a unique fixed point in the metric space $\left\{\pi \in L^{\delta}\left(0,2 \bar{t} ; W^{1, d}(\Omega)\right) \mid \pi=\right.$ $\hat{y}$ on $] 0, \bar{t}[\}$. We want to estimate the solution in $L^{\delta}\left(0,2 \bar{t} ; W^{1, d}(\Omega)\right)$. Let $\left(\xi_{n}\right)_{n}$ be the sequence defined by:

$$
\xi_{0}=\hat{y} \text { on }(0, \bar{t}), \xi_{0}=0 \text { on }(\bar{t}, 2 \bar{t}), \quad \xi_{n}=y_{\xi_{n-1}} \text { on }(0,2 \bar{t}) .
$$

Then $\left(\xi_{n}\right)_{n}$ converges to $y$ in $L^{\delta}\left(0,2 \bar{t} ; W^{1, d}(\Omega)\right)$. From the properties of the fixed point, we have

$$
\left\|y_{\xi_{n}}-y_{\xi_{0}}\right\|_{L^{\delta}\left(0,2 \bar{t} ; W^{1, d}(\Omega)\right)} \leq\left\|y_{\xi_{0}}-\xi_{0}\right\|_{L^{\delta}\left(0,2 \bar{t} ; W^{1, d}(\Omega)\right)} .
$$

Since $y_{\xi_{0}}$ is the solution to the equation

$$
\frac{\partial y}{\partial t}+A y=-\vec{V} \cdot \nabla \xi_{0} \text { in } Q_{2 \bar{t}}, \quad \frac{\partial y}{\partial n_{A}}=u \delta_{\gamma} \text { on } \Sigma_{2 \bar{t}}, \quad y(0)=0 \text { in } \Omega
$$

then

$$
\left\|y_{\xi_{0}}\right\|_{L^{\delta}\left(0,2 \bar{t} ; W^{1, d}(\Omega)\right)} \leq K\left(1+\|\vec{V}\|_{L^{\tilde{m}}\left(0, T ;\left(L^{m}(\Omega)\right)^{N}\right)}\right)\|u\|_{L^{q}\left(0, T ; L^{\sigma}(\gamma)\right)} .
$$

Therefore, we have:

$$
\|y\|_{L^{\delta}\left(0,2 \bar{t} ; W^{1, d}(\Omega)\right)} \leq K\left(1+\|\vec{V}\|_{L^{\tilde{m}}\left(0, T ;\left(L^{m}(\Omega)\right)^{N}\right)}\right)\|u\|_{L^{q}\left(0, T ; L^{\sigma}(\gamma)\right)} .
$$

4 - Estimate of the global solution in $L^{\delta}\left(0, T ; W^{1, d}(\Omega)\right)$. By induction, it is easy to prove that

$$
\|y\|_{L^{\delta}\left(0, T ; W^{1, d}(\Omega)\right)} \leq K_{n}\left(1+\|\vec{V}\|_{\left.L^{\tilde{m}}\left(0, T ; L^{m}(\Omega)\right)^{N}\right)}+\ldots+\|\vec{V}\|_{L^{\tilde{m}}\left(0, T ;\left(L^{m}(\Omega)\right)^{N}\right)}^{n-1}\right)\|u\|_{L^{q}\left(0, T ; L^{\sigma}(\gamma)\right)}
$$

where $n=\left[\frac{T}{t}\right]+1$, and where $K_{n}$ depends on $n$ (observe that $n$ depends on $\bar{t}$, and $\bar{t}$ depends on $\vec{V}$ ). Therefore, there exists a constant $\tilde{C}$ depending on $\vec{V}$ and $T$, such that

$$
\|y\|_{L^{\delta}\left(0, T ; W^{1, d}(\Omega)\right)} \leq \tilde{C}\|u\|_{L^{q}\left(0, T ; L^{\sigma}(\gamma)\right)} .
$$

5 - Estimate in $L^{\infty}\left(0, T ; L^{r}(\Omega)\right)$. Observe that $y=y_{1}+y_{2}$, where $y_{1}$ and $y_{2}$ are the solutions to the equations:

$$
\begin{gathered}
\frac{\partial y_{1}}{\partial t}+A y_{1}=0 \text { in } Q, \quad \frac{\partial y_{1}}{\partial n_{A}}=u \delta_{\gamma} \text { on } \Sigma, \quad y_{1}(0)=0 \text { in } \Omega, \\
\frac{\partial y_{2}}{\partial t}+A y_{2}=-\vec{V} \cdot \nabla y \text { in } Q, \quad \frac{\partial y_{2}}{\partial n_{A}}=0 \text { on } \Sigma, \quad y_{2}(0)=0 \text { in } \Omega .
\end{gathered}
$$

Due to Proposition 2.1, $y_{1}$ belongs to $L^{\infty}\left(0, T ; L^{r}(\Omega)\right)$ for every $1 \leq r<\inf \left\{\frac{N}{N-\frac{D}{N-D}-\frac{2}{q^{\prime}}}, \frac{N}{N-\frac{D}{\sigma^{r}}-\frac{2}{q^{\prime}}}\right\} \leq$ $\inf \left\{\frac{N-D}{N-D-\frac{2}{q^{\prime}}}, \frac{N}{N-\frac{D}{\sigma^{\prime}}-\frac{2}{q^{\prime}}}\right\}$.

If $\sigma<\frac{N-D}{N-D-1}$, then inf $\left\{\frac{N}{N-\frac{D}{N-D}-\frac{2}{q^{\prime}}}, \frac{N}{N-\frac{D}{\sigma^{\prime}}-\frac{2}{q^{\prime}}}\right\}=\frac{N}{N-\frac{D}{\sigma^{\prime}}-\frac{2}{q^{\prime}}}$, and $\sigma<\frac{N}{N-\frac{D}{\sigma^{\prime}}-1}$. Let $r$ satisfy $\sigma<\frac{N}{N-\frac{D}{\sigma^{\prime}}-1}<$ $r<\frac{N}{N-\frac{D}{\sigma^{\prime}}-\frac{2}{q^{\prime}}}$. Observe that $\frac{1}{r}<\frac{1}{m}+\frac{1}{\sigma}, \frac{1}{q}+\frac{N}{2 r}-\frac{N}{2 m}<\frac{N}{2 r}+\frac{1}{2}$, and $\frac{1}{q}+\frac{1}{2}\left(N-\frac{D}{\sigma^{\prime}}-1\right)=\frac{1}{2}\left(N-\frac{D}{\sigma^{\prime}}-\frac{2}{q^{\prime}}\right)+\frac{1}{2}<\frac{N}{2 r}+\frac{1}{2}$. Therefore, there exists $d$ satisfying

$$
\sup \left\{\frac{N}{2 r}-\frac{N}{2 m}, \frac{1}{2}\left(N-\frac{D}{\sigma^{\prime}}-1\right)\right\}<\frac{N}{2 d}<\inf \left\{\frac{N}{2 \sigma}, \frac{N}{2 r}+\frac{1}{2}-\frac{1}{q}\right\} .
$$


Since $\frac{1}{2}\left(N-\frac{D}{\sigma^{\prime}}-1\right)<\frac{N}{2 d}$, applying (17), we deduce that $y$ belongs to $L^{q}\left(0, T ; W^{1, d}(\Omega)\right)$. Set $\frac{1}{\ell}=\frac{1}{\tilde{m}}+\frac{1}{q}$, and $\frac{1}{\ell}=\frac{1}{m}+\frac{1}{d}$. Then $\tilde{\ell}>1, \ell>1$, and $\vec{V} \cdot \nabla y$ belongs to $L^{\tilde{\ell}}\left(0, T ; L^{\ell}(\Omega)\right)$. Since $\frac{1}{r}-\frac{1}{m}<\frac{1}{d}$, we have $r \geq \ell$. From $\frac{N}{2 d}<\frac{N}{2 r}+\frac{1}{2}-\frac{1}{q}$ and $\frac{1}{\tilde{m}}+\frac{N}{2 m} \leq \frac{1}{2}$, it follows that $\frac{1}{\ell}+\frac{N}{2 \ell}<\frac{N}{2 r}+1$. Due to Proposition 2.6, we deduce that $y_{2}$ belongs to $L^{\infty}\left(0, T ; L^{r}(\Omega)\right)$ for all $r<\frac{N}{N-\frac{D}{\sigma^{\prime}}-\frac{2}{q^{\prime}}}$.

If $\sigma \geq \frac{N-D}{N-D-1}$, then $\inf \left\{\frac{N}{N-\frac{D}{N-D}-\frac{2}{q^{\prime}}}, \frac{N}{N-\frac{D}{\sigma^{\prime}}-\frac{2}{q^{\prime}}}\right\}=\frac{N}{N-\frac{D}{N-D}-\frac{2}{q^{\prime}}}$. Let $r$ satisfy $1<r<\frac{N}{N-\frac{D}{(N-D)}-\frac{2}{q^{\prime}}}$. We have $q \geq 2, \frac{N-D-1}{N-D}<\frac{1}{m^{\prime}}$, and $\frac{N(N-D-1)}{(N-D)}<\frac{N}{r}+1-\frac{2}{q}$. Thus there exists $d$ satisfying

$$
\sup \left\{\frac{N}{2 r}-\frac{N}{2 m}, \frac{N(N-D-1)}{2(N-D)}\right\}<\frac{N}{2 d}<\inf \left\{\frac{N}{2 m^{\prime}}, \frac{N}{2 r}+\frac{1}{2}-\frac{1}{q}\right\} \text {. }
$$

Applying (17), we deduce that $y$ belongs to $L^{q}\left(0, T ; W^{1, d}(\Omega)\right)$. Set $\frac{1}{\ell}=\frac{1}{\tilde{m}}+\frac{1}{q}$, and $\frac{1}{\ell}=\frac{1}{m}+\frac{1}{d}$. Then $\tilde{\ell}>1$, $\ell>1$, and $\vec{V} \cdot \nabla y$ belongs to $L^{\tilde{\ell}}\left(0, T ; L^{\ell}(\Omega)\right)$. Since $\frac{1}{r}-\frac{1}{m}<\frac{1}{d}$, we have $r \geq \ell$. From $\frac{N}{2 d}<\frac{N}{2 r}+\frac{1}{2}-\frac{1}{q}$ and $\frac{1}{\tilde{m}}+\frac{N}{2 m} \leq \frac{1}{2}$, it follows that $\frac{1}{\bar{\ell}}+\frac{N}{2 \ell}<\frac{N}{2 r}+1$. Due to Proposition 2.6, we deduce that $y_{2}$ belongs to $L^{\infty}\left(0, T ; L^{r}(\Omega)\right)$ for all $r<\frac{N}{N-\frac{D}{(N-D)}-\frac{2}{q^{\prime}}}$.

Using the same argument as in the proof of Proposition 2.1 in [10], we can prove that $y$ belongs to $C\left([0, T] ; L_{w}^{r}(\Omega)\right)$ for every $1 \leq r<\inf \left\{\frac{N}{N-\frac{D}{N-D}-\frac{2}{q^{\prime}}}, \frac{N}{N-\frac{D}{\sigma^{\prime}}-\frac{2}{q^{\prime}}}\right\}$.

6 - Uniqueness. If we consider the equation

$$
\frac{\partial y}{\partial t}+A y+\vec{V} \cdot \nabla y=0 \text { in } Q, \quad \frac{\partial y}{\partial n_{A}}=0 \text { on } \Sigma, \quad y(0)=0 \text { in } \Omega,
$$

we can apply the above fixed point method to prove that $y \equiv 0$ is the unique solution to this equation in $L^{\hat{\delta}}\left(0, T ; W^{1, \hat{d}}(\Omega)\right)$, for all $(\hat{\delta}, \hat{d})$ obeying $\tilde{m}^{\prime}<\hat{\delta} \leq q$, and all $m^{\prime}<\hat{d}<\inf \left\{\frac{N}{N-\frac{D}{\sigma^{\prime}}-1}, \frac{N-D}{N-D-1}\right\}$.

Proposition 2.8. Consider the equation

$$
\frac{\partial y}{\partial t}+A y+\vec{V} \cdot \nabla y=0 \text { in } Q, \quad \frac{\partial y}{\partial n_{A}}=0 \text { on } \Sigma, \quad y(0)=y_{0} \text { in } \Omega,
$$

where $y_{0}$ belongs to $L^{\rho}(\Omega)$ with $\rho=\frac{N}{N-\frac{D}{\sigma^{\prime}}-\frac{2}{q^{\prime}}}$. The mapping that associates the solution $y$ with $y_{0}$ is continuous from $L^{\rho}(\Omega)$ into $L^{\delta_{2}}\left(0, T ; W^{1, d_{2}}(\Omega)\right)$ for every $\left(\delta_{2}, d_{2}\right)$ satisfying

$$
\tilde{m}^{\prime}<\delta_{2}<2, \quad \rho \leq d_{2}<\frac{N \rho}{N-\rho}, \quad 1+\frac{N}{2 \rho}<\frac{1}{\delta_{2}}+\frac{N}{2 d_{2}}+\frac{1}{2} .
$$

The mapping that associates the solution $y$ with $y_{0}$ is continuous from $L^{\rho}(\Omega)$ into $L^{\infty}\left(0, T ; L^{r}(\Omega)\right)$ for every $1 \leq r<\rho$. Moreover, $y$ belongs to $C\left([0, T] ; L^{r}(\Omega)\right)$ for every $1 \leq r<\rho$.

Proof. 1 - We still use a fixed point method. Let $\left(\delta_{2}, d_{2}\right)$ be a pair satisfying $(24)$. Let $\xi \in L^{\delta_{2}}\left(0, \bar{t} ; W^{1, d_{2}}(\Omega)\right)$, and $y_{\xi}$ be the solution to the equation:

$$
\frac{\partial y_{\xi}}{\partial t}+A y_{\xi}=-\vec{V} \cdot \nabla \xi \text { in } Q_{\bar{t}}, \quad \frac{\partial y_{\xi}}{\partial n_{A}}=0 \text { on } \Sigma_{\bar{t}}, \quad y_{\xi}(0)=y_{0} \text { in } \Omega
$$


Then $y_{\xi}=\hat{y}+\tilde{y}$, where $\hat{y}$ and $\tilde{y}$ satisfy the following equations:

$$
\begin{gathered}
\frac{\partial \hat{y}}{\partial t}+A \hat{y}=0 \text { in } Q_{\bar{t}}, \quad \frac{\partial \hat{y}}{\partial n_{A}}=0 \text { on } \Sigma_{\bar{t}}, \quad \hat{y}(0)=y_{0} \text { in } \Omega, \\
\frac{\partial \tilde{y}}{\partial t}+A \tilde{y}=-\vec{V} \cdot \nabla \xi \text { in } Q_{\bar{t}}, \quad \frac{\partial \tilde{y}}{\partial n_{A}}=0 \text { on } \Sigma_{\bar{t}}, \quad \tilde{y}(0)=0 \text { in } \Omega .
\end{gathered}
$$

From Proposition 2.2, it follows that $\hat{y} \in L^{\delta_{2}}\left(0, \bar{t} ; W^{1, d_{2}}(\Omega)\right)$. Since $\delta_{2}>\tilde{m}^{\prime}$, and $d_{2}>m^{\prime}$, then $\frac{\tilde{m} \delta_{2}}{\tilde{m}+\delta_{2}}>$ $1, \frac{m d_{2}}{m+d_{2}}>1$, and $\vec{V} \cdot \nabla \xi$ belongs to $L^{\frac{\tilde{m} \delta_{2}}{m+\delta_{2}}}\left(0, \bar{t} ; L^{\frac{m d_{2}}{m+d_{2}}}(\Omega)\right)$. Using Proposition 2.6 for $k=1$, we deduce that $\tilde{y} \in L^{\delta_{2}}\left(0, \bar{t} ; W^{1, d_{2}}(\Omega)\right)$. Thus $y_{\xi} \in L^{\delta_{2}}\left(0, \bar{t} ; W^{1, d_{2}}(\Omega)\right)$.

We prove that the mapping $\xi \mapsto y_{\xi}$ admits a fixed point in $L^{\delta_{2}}\left(0, \bar{t} ; W^{1, d_{2}}(\Omega)\right)$ for some $\bar{t}>0$. Let $\left(\xi_{1}, \xi_{2}\right)$ belong to $L^{\delta_{2}}\left(0, \bar{t} ; W^{1, d_{2}}(\Omega)\right)$. Still with Proposition 2.6, we have:

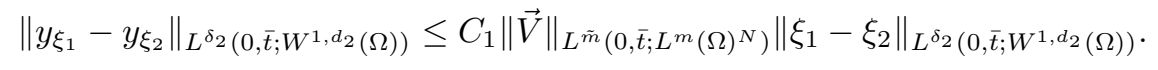

The mapping $t \mapsto C_{1}^{\tilde{m}} \int_{0}^{t}\|\vec{V}(\tau)\|_{L^{m}(\Omega)^{N}}^{\tilde{m}} \mathrm{~d} \tau$ is absolutely continuous, there exists $\bar{t}$ depending on $\vec{V}$, such that $C_{1}\left(\int_{t}^{\min \{t+\bar{t}, T\}}\|\vec{V}(\tau)\|_{L^{m}(\Omega)^{N}}^{\tilde{m}} \mathrm{~d} \tau\right)^{\frac{1}{m}} \leq C=\frac{1}{2}$ for all $t \in[0, T]$. Therefore the mapping $\xi \mapsto y_{\xi}$ is a contraction in $L^{\delta_{2}}\left(0, \bar{t} ; W^{1, d_{2}}(\Omega)\right)$, and it admits a unique fixed point in this space. Next, we can prove the existence of a unique global solution in $L^{\delta_{2}}\left(0, T ; W^{1, d_{2}}(\Omega)\right)$ as in the proof of Proposition 2.7.

2 - Let $\frac{\rho m}{\rho+m} \leq r<\rho$. Observe that $y=y_{1}+y_{2}$, where $y_{1}$ and $y_{2}$ are the solutions to the equations:

$$
\begin{gathered}
\frac{\partial y_{1}}{\partial t}+A y_{1}=0 \text { in } Q, \quad \frac{\partial y_{1}}{\partial n_{A}}=0 \text { on } \Sigma, \quad y_{1}(0)=y_{0} \text { in } \Omega, \\
\frac{\partial y_{2}}{\partial t}+A y_{2}=-\vec{V} \cdot \nabla y \text { in } Q, \quad \frac{\partial y_{2}}{\partial n_{A}}=0 \text { on } \Sigma, \quad y_{2}(0)=0 \text { in } \Omega .
\end{gathered}
$$

Due to Proposition 2.4, $y_{1}$ belongs to $L^{\infty}\left(0, T ; L^{\rho}(\Omega)\right)$. Since $\frac{1}{2}+\frac{N}{2 \rho}-\frac{N}{2 r}+\frac{N}{2 m}<\frac{N}{2 m}+\frac{1}{2}$, we can choose $\tilde{m}^{\prime}<\delta_{2}<2$ such that $\frac{1}{2}+\frac{N}{2 \rho}-\frac{N}{2 r}+\frac{N}{2 m}<\frac{1}{\delta_{2}}<\frac{N}{2 m}+\frac{1}{2} \leq \frac{1}{\tilde{m}^{\prime}}$. We take $\frac{1}{d_{2}}=\frac{1}{r}-\frac{1}{m}$. Then $\left(\delta_{2}, d_{2}\right)$ satisfies (24), and $y$ belongs to $L^{\delta_{2}}\left(0, T ; W^{1, d_{2}}(\Omega)\right)$. Moreover $\frac{1}{\delta_{2}}+\frac{N}{2 d_{2}}<\frac{N}{2 r}+1-\frac{1}{\tilde{m}}-\frac{N}{2 m}$. Set $\frac{1}{\ell}=\frac{1}{\tilde{m}}+\frac{1}{\delta_{2}}$, then $\tilde{\ell}>1$, and $\vec{V} \cdot \nabla y$ belongs to $L^{\tilde{\ell}}\left(0, T ; L^{r}(\Omega)\right)$. Due to Proposition 2.6, we deduce that $y_{2}$ belongs to $C\left([0, T] ; L^{r}(\Omega)\right)$.

\section{Control problem $\left(\mathrm{P}_{1}\right)$}

\subsection{Existence of solutions to problem $\left(\mathbf{P}_{\mathbf{1}}\right)$}

Theorem 3.1. Assume that hypotheses (A1) to (A7) are satisfied. Suppose that there exist $(\delta, d)$ satisfying (17), and $\left(\delta_{2}, d_{2}\right)$ satisfying (24), such that $\kappa \leq \delta, \kappa \leq d, \kappa \leq \delta_{2}$, and $\kappa \leq d_{2}$. Suppose in addition that $\theta<$ $\inf \left\{\frac{N}{N-\frac{D}{N-D}-\frac{2}{q^{\prime}}}, \frac{N}{N-\frac{D}{\sigma^{\prime}}-\frac{2}{q^{\prime}}}\right\}$. Then the control problem $\left(P_{1}\right)$ admits solutions.

Proof. Let $\left(u_{n}\right)_{n}$ be a minimizing sequence in $K_{U}$. Then $\left(u_{n}\right)_{n}$ is bounded in $L^{q}\left(0, T ; L^{\sigma}(\gamma)\right)$. We can suppose that $\left(u_{n}\right)_{n}$ converges to some $\bar{u}$ weakly-star in $L^{q}\left(0, T ; L^{\sigma}(\gamma)\right)$. Since $K_{U}$ is convex and closed in $L^{q}\left(0, T ; L^{\sigma}(\gamma)\right)$, then $\bar{u} \in K_{U}$. Due to Propositions 2.7 and 2.8 , the sequence $\left(y_{n}\right)_{n}$ is bounded in $L^{\delta}(0, T$; $\left.W^{1, d}(\Omega)\right)+L^{\delta_{2}}\left(0, T ; W^{1, d_{2}}(\Omega)\right)$ for all $(\delta, d)$ satisfying $(17)$, and all $\left(\delta_{2}, d_{2}\right)$ satisfying $(24)$. Therefore we can suppose that $\left(y_{n}\right)_{n}$ converges to some $\bar{y}$ for the weak topology of $L^{\delta}\left(0, T ; W^{1, d}(\Omega)\right)+L^{\delta_{2}}\left(0, T ; W^{1, d_{2}}(\Omega)\right)$ for all $(\delta, d)$ satisfying $(17)$, and all $\left(\delta_{2}, d_{2}\right)$ satisfying $(24)$. We can easily verify that $\bar{y}$ is the solution to equation (1) corresponding to $\bar{u}$. Since $\kappa \leq \delta, \kappa \leq d, \kappa \leq \delta_{2}, \kappa \leq d_{2}$ for some $(\delta, d)$ satisfying (17), and some $\left(\delta_{2}, d_{2}\right)$ satisfying $(24),\left(y_{n}\right)_{n}$ converges to $\bar{y}$ for the weak topology of $L^{\kappa}\left(0, T ; W^{1, \kappa}(\Omega)\right)$. Moreover, due to 
Propositions 2.8 and 2.7, we can prove that $\left(y_{n}(T)\right)_{n}$ is bounded in $L^{\theta}(\Omega)$, and that $\left(y_{n}(T)\right)_{n}$ converges to $\bar{y}(T)$ for the weak topology of $L^{\theta}(\Omega)$. By classical arguments, we can prove that $(\bar{u}, \bar{y})$ is a solution of $\left(P_{1}\right)$.

\subsection{Regularity results for the adjoint equation}

To study the control problem $\left(P_{1}\right)$, we look for solutions to equation $(1)$ belonging to $L^{\kappa}\left(0, T ; W^{1, \kappa}(\Omega)\right)$. Therefore we must have $\kappa \leq \delta, \kappa \leq d, \kappa \leq \delta_{2}, \kappa \leq d_{2}$ for some $(\delta, d)$ satisfying (17), and some $\left(\delta_{2}, d_{2}\right)$ satisfying (24). Observe that if $\sigma \geq \frac{N-D}{N-D-1}$, and if we take $\delta=q$, then (17) is satisfied for all $1 \leq d<\frac{N-D}{N-D-1}$. If we take $d_{2}=\rho$, then (24) is satisfied for all $1 \leq \delta_{2}<2$. Due to these observations, to simplify the calculations, throughout the sequel we make the following additional assumptions.

(A8) $\quad \sigma \geq \frac{N-D}{N-D-1}, \quad q \geq \sigma$. The function $V_{d}$ belongs to $L^{q}\left(0, T ;\left(L^{\frac{N-D}{N-D-1}}(\Omega)\right)^{N}\right)+\left(L^{2}\left(0, T ;\left(L^{\rho}(\Omega)\right)^{N}\right)\right.$. The function $y_{d}$ belongs to $L^{\hat{r}}(\Omega)$, where $\hat{r}=\frac{N}{\left(N-\frac{D}{N-D}-\frac{2}{q^{\prime}}\right)}$.

We consider the following terminal boundary value problem

$$
-\frac{\partial p}{\partial t}+A p-\vec{V} \cdot \nabla p=-\operatorname{div} \vec{h} \text { in } Q, \quad \frac{\partial p}{\partial n_{A}}=\vec{h} \cdot \vec{n} \text { on } \Sigma, \quad p(T)=p_{T} \text { in } \Omega .
$$

When $\vec{h} \cdot \vec{n}$ is not defined, equation (26) is a formal writing for the variational equation:

$$
\int_{Q}\left(p \frac{\partial y}{\partial t}+\sum_{i, j=1}^{N} a_{i j} D_{j} p D_{i} y+a_{0} p y-\vec{V} \cdot \nabla p y\right) \mathrm{d} x \mathrm{~d} t=\int_{Q} \vec{h} \cdot \nabla y \mathrm{~d} x \mathrm{~d} t+\int_{\Omega} y(T) p_{T} \mathrm{~d} x,
$$

for all $y \in C^{1}(\bar{Q})$ such that $y(0)=0$. If $u$ is a solution of $\left(P_{1}\right)$, if $y_{u}$ is the solution of (1) corresponding to $u$, if we set $\vec{h}=\kappa C_{Q}\left|\nabla y_{u}-V_{d}\right|^{\kappa-2}\left(\nabla y_{u}-V_{d}\right)$, and $p_{T}=\theta C_{\Omega}\left|y_{u}(T)-y_{d}\right|^{\theta-2}\left(y_{u}(T)-y_{d}\right)$, then equation (26) corresponds to the adjoint equation for $\left(P_{1}\right)$ associated with $\left(u, y_{u}\right)$. Due to Propositions 2.7, 2.8, assumption (A8), and Lemma 4.1 in [21], the function $\vec{h}=\kappa C_{Q}\left|\nabla y_{u}-V_{d}\right|^{\kappa-2}\left(\nabla y_{u}-V_{d}\right)$ belongs to $L^{\frac{q}{\kappa-1}}\left(0, T ;\left(L^{\frac{d}{\kappa-1}}(\Omega)\right)^{N}\right)+$ $L^{\frac{\delta_{2}}{\kappa-1}}\left(0, T ;\left(L^{\frac{\rho}{\kappa-1}}(\Omega)\right)^{N}\right.$ for all $1 \leq d<\frac{N-D}{N-D-1}$ and all $1 \leq \delta_{2}<2$. The function $p_{T}=\theta C_{\Omega} \mid y_{u}(T)-$ $\left.y_{d}\right|^{\theta-2}\left(y_{u}(T)-y_{d}\right)$ belongs to $L^{\epsilon}(\Omega)$ for all $1 \leq \epsilon<\frac{N}{\left(N-\frac{D}{N-D}-\frac{2}{q^{\prime}}\right)(\theta-1)}$. This is the reason why we now study the regularity of the solution $p$ to equation (26) when $\vec{h}$ and $p_{T}$ satisfy such conditions. In particular, to prove the optimality conditions for $\left(P_{1}\right)$, we establish that the trace of $p$ on $\left.\gamma \times\right] 0, T$ [ belongs to $L^{q^{\prime}}\left(0, T ; L^{\sigma^{\prime}}(\gamma)\right)$. We study equation (26) for $p_{T} \equiv 0$ and $\vec{h} \not \equiv 0$ in Theorem 3.2 and Theorem 3.3 , for $\vec{h} \equiv 0$ and $p_{T} \not \equiv 0$ in Theorem 3.3. We summarize these results in Theorem 3.4.

Theorem 3.2. Suppose that $\vec{h}$ belongs to $L^{\tilde{\eta}}\left(0, T ;\left(L^{\eta}(\Omega)\right)^{N}\right)$ with $\tilde{\eta}>\tilde{m}^{\prime}$ and $\eta>m^{\prime}$. Consider the equation:

$$
-\frac{\partial p}{\partial t}+A p-\vec{V} \cdot \nabla p=-\operatorname{div} \vec{h} \text { in } Q, \quad \frac{\partial p}{\partial n_{A}}=\vec{h} \cdot \vec{n} \text { on } \Sigma, \quad p(T)=0 \text { in } \Omega .
$$

Equation (27) admits a unique weak solution in $L^{\tilde{\eta}}\left(0, T ; W^{1, \eta}(\Omega)\right)$. If $\tilde{\eta} \geq q^{\prime}$ and $\eta>N-D$, then the trace of $p$ on $\gamma \times] 0, T\left[\right.$ belongs to $L^{q^{\prime}}\left(0, T ; L^{\sigma^{\prime}}(\gamma)\right)$.

Proof. To study equation (27), we still use a fixed point method as in the proof of Proposition 2.7. Let $\xi$ belong to $L^{\tilde{\eta}}\left(T-\bar{t}, T ; W^{1, \eta}(\Omega)\right)$, and let $p_{\xi}$ be the solution of the equation:

$$
\left.-\frac{\partial p}{\partial t}+A p=-\operatorname{div} \vec{h}+\vec{V} \cdot \nabla \xi \text { in } \Omega \times\right] T-\bar{t}, T\left[, \quad \frac{\partial p}{\partial n_{A}}=\vec{h} \cdot \vec{n} \text { on } \Gamma \times\right] T-\bar{t}, T[, \quad p(T)=0 \text { in } \Omega .
$$

Set $\frac{1}{\ell}=\frac{1}{\tilde{m}}+\frac{1}{\tilde{\eta}}, \frac{1}{\ell}=\frac{1}{m}+\frac{1}{\eta}$. Then $\tilde{\ell}>1, \ell>1$, and $\vec{V} \cdot \nabla \xi$ belongs to $L^{\tilde{\ell}}\left(T-\bar{t}, T ; L^{\ell}(\Omega)\right)$. From Proposition 2.5, and Proposition 2.6 for $k=1$, it follows that $p_{\xi}$ belongs to $L^{\tilde{\eta}}\left(T-\bar{t}, T ; W^{1, \eta}(\Omega)\right)$. 
Let $\xi_{1}$ and $\xi_{2}$ belong to $L^{\tilde{\eta}}\left(T-\bar{t}, T ; W^{1, \eta}(\Omega)\right)$. Still with Proposition 2.6, we have:

$$
\left\|p_{\xi_{1}}-p_{\xi_{2}}\right\|_{L^{\tilde{\eta}}\left(T-\bar{t}, T ; W^{1, \eta}(\Omega)\right)} \leq C\|\vec{V}\|_{L^{\tilde{m}}\left(T-\bar{t}, T ;\left(L^{m}(\Omega)\right)^{N}\right)}\left\|\xi_{1}-\xi_{2}\right\|_{L^{\tilde{\eta}}\left(T-\bar{t}, T ; W^{1, \eta}(\Omega)\right)},
$$

where $C$ is independent of $\bar{t}$. For $\bar{t}>0$ small enough, we have $C\|\vec{V}\|_{L^{\tilde{m}}\left(T-\bar{t}, T ;\left(L^{m}(\Omega)\right)^{N}\right)}<1$.

Therefore, there exists $\bar{t}>0$ such that the mapping $\xi \mapsto p_{\xi}$ is a contraction in the Banach space $L^{\tilde{\eta}}(T-\bar{t}, T$; $W^{1, \eta}(\Omega)$ ), and it admits a fixed point. As in the proof of Proposition 2.7, we can prove that equation (27) admits a unique global solution in $L^{\tilde{\eta}}\left(0, T ; W^{1, \eta}(\Omega)\right)$.

If $\tilde{\eta} \geq q^{\prime}$ and $\eta>N-D$, using Proposition 2.3, the trace of $p$ on $\left.\gamma \times\right] 0, T\left[\right.$ belongs to $L^{q^{\prime}}\left(0, T ; L^{\eta}(\gamma)\right)$. Since $\eta>N-D \geq \sigma^{\prime}$, the trace of $p$ on $\left.\gamma \times\right] 0, T$ [ belongs to $L^{q^{\prime}}\left(0, T ; L^{\sigma^{\prime}}(\gamma)\right)$.

Remark. Since $N-D \geq \frac{N}{N-1}>m^{\prime}$, the condition $\eta>N-D$ is stronger than $\eta>m^{\prime}$.

Theorem 3.3. Consider the equation

$$
-\frac{\partial p}{\partial t}+A p-\vec{V} \cdot \nabla p=0 \text { in } Q, \quad \frac{\partial p}{\partial n_{A}}=0 \text { on } \Sigma, \quad p(T)=p_{T} \text { in } \Omega,
$$

where $p_{T}$ belongs to $L^{\epsilon}(\Omega)$, with $\epsilon$ satisfying $\frac{N}{2 \epsilon}<\frac{1}{\tilde{m}^{\prime}}+\frac{D}{2(N-D)}$ and $\frac{N}{2 \epsilon}<\frac{1}{q^{\prime}}+\frac{D}{2(N-D)}$. Equation (28) admits a unique solution in $L^{\tilde{k}}\left(0, T ; W^{1, k}(\Omega)\right)$ for some $(\tilde{k}, k)$ satisfying

$$
\tilde{k}>\tilde{m}^{\prime}, \quad k \geq \epsilon, \quad k>N-D>m^{\prime}, \quad \frac{N}{2 \epsilon}+\frac{1}{2}<\frac{1}{\tilde{k}}+\frac{N}{2 k} .
$$

Moreover, the trace of $p$ on $\gamma \times] 0, T\left[\right.$ belongs to $L^{q^{\prime}}\left(0, T ; L^{\sigma^{\prime}}(\gamma)\right)$.

Proof. We distinguish the cases $\epsilon \leq N-D$ and $\epsilon>N-D$.

1 - First consider the case $\epsilon \leq N-D$. From the inequality $\frac{N}{2(N-D)} \leq \frac{N}{2 \epsilon}<\frac{1}{q^{\prime}}+\frac{D}{2(N-D)}$, it follows that $q^{\prime}<2$. There exists $\max \left(q^{\prime}, \tilde{m}^{\prime}\right)<\tilde{k}<2$ such that

$$
\frac{N}{2 \epsilon}+1<\frac{1}{\tilde{k}}+\frac{D}{2(N-D)}+\frac{1}{2}+\frac{1}{2}=\frac{1}{\tilde{k}}+\frac{N}{2(N-D)}+\frac{1}{2} .
$$

Therefore there exists $k>N-D>m^{\prime}$ such that

$$
k \geq \epsilon, \quad \frac{N}{2 \epsilon}+1<\frac{N}{2 k}+\frac{1}{\tilde{k}}+\frac{1}{2}<\frac{N}{2(N-D)}+\frac{1}{\tilde{k}}+\frac{1}{2} .
$$

To study equation (28), we still use a fixed point argument. Let $\xi$ belong to $L^{\tilde{k}}\left(T-\bar{t}, T ; W^{1, k}(\Omega)\right)$, and $p_{\xi}$ be the solution of the equation:

$$
\left.-\frac{\partial p}{\partial t}+A p=\vec{V} \cdot \nabla \xi \text { in } \Omega \times\right] T-\bar{t}, T\left[, \quad \frac{\partial p}{\partial n_{A}}=0 \text { on } \Gamma \times\right] T-\bar{t}, T\left[, \quad p(T)=p_{T} \text { in } \Omega .\right.
$$

Set $\frac{1}{\tilde{\ell}}=\frac{1}{\tilde{m}}+\frac{1}{\tilde{k}}, \frac{1}{\ell}=\frac{1}{m}+\frac{1}{k}$. Observe that $\vec{V} \cdot \nabla \xi$ belongs to $L^{\tilde{\ell}}\left(T-\bar{t}, T ; L^{\ell}(\Omega)\right)$, with $\tilde{\ell}>1, \ell>1$. From Proposition 2.6, and Proposition 2.4 with $\alpha=0$, it follows that $p_{\xi} \in L^{\tilde{k}}\left(T-\bar{t}, T ; W^{1, k}(\Omega)\right)$.

Let $\xi_{1}$ and $\xi_{2}$ belong to $L^{\tilde{k}}\left(T-\bar{t}, T ; W^{1, k}(\Omega)\right)$. Still with Proposition 2.6, we have:

$$
\left\|p_{\xi_{1}}-p_{\xi_{2}}\right\|_{L^{\tilde{k}}\left(T-\bar{t}, T ; W^{1, k}(\Omega)\right)} \leq C\|\vec{V}\|_{L^{\tilde{m}}\left(T-\bar{t}, T ; L^{m}(\Omega)\right)}\left\|\xi_{1}-\xi_{2}\right\|_{L^{\tilde{k}}\left(T-\bar{t}, T ; W^{1, k}(\Omega)\right)},
$$


where $C$ is independent of $\bar{t}$. There exists $\bar{t}>0$ such that the mapping $\xi \mapsto p_{\xi}$ is a contraction in the Banach space $L^{\tilde{k}}\left(T-\bar{t}, T ; W^{1, k}(\Omega)\right)$, and it admits a fixed point. As in the proof of Proposition 2.7, we can prove that equation (28) admits a unique solution in $L^{\tilde{k}}\left(0, T ; W^{1, k}(\Omega)\right)$.

Since $\tilde{k} \geq q^{\prime}$, and $k>N-D \geq \sigma^{\prime}$, the trace of $p$ on $\left.\gamma \times\right] 0, T$ [ belongs to $L^{q^{\prime}}\left(0, T ; L^{\sigma^{\prime}}(\gamma)\right)$.

2 - Now we study the case $\epsilon>N-D$. We choose $\frac{N-D}{\epsilon}<r<1$, for example we can set $r=\frac{1}{2}\left(1+\frac{N-D}{\epsilon}\right)$. We choose

$$
\frac{1}{2}<\frac{1}{\tilde{k}}<\min \left(\frac{1}{\tilde{m}^{\prime}}, 1-\frac{r}{2}\right)
$$

Still using a fixed point method, we prove that equation (28) admits a unique solution in $L^{\tilde{k}}\left(0, T ; W^{1, \epsilon}(\Omega)\right) \cap$ $L^{2}\left(0, T ; W^{r, \epsilon}(\Omega)\right)$. Let $\xi$ belong to $L^{\tilde{k}}\left(T-\bar{t}, T ; W^{1, \epsilon}(\Omega)\right) \cap L^{2}\left(T-\bar{t}, T ; W^{r, \epsilon}(\Omega)\right)$ be the solution of the equation:

$$
\left.-\frac{\partial p}{\partial t}+A p=\vec{V} \cdot \nabla \xi \text { in } \Omega \times\right] T-\bar{t}, T\left[, \quad \frac{\partial p}{\partial n_{A}}=0 \text { on } \Gamma \times\right] T-\bar{t}, T\left[, \quad p(T)=p_{T} \text { in } \Omega .\right.
$$

Set $\frac{1}{\ell}=\frac{1}{\tilde{m}}+\frac{1}{k}, \frac{1}{\ell}=\frac{1}{m}+\frac{1}{\epsilon}$. Then $\vec{V} \cdot \nabla \xi$ belongs to $L^{\tilde{\ell}}\left(T-\bar{t}, T ; L^{\ell}(\Omega)\right)$, with $\tilde{\ell}>1, \ell>1$. Due to (31), we have

and

$$
\frac{N}{2 \epsilon}+1<\frac{N}{2 \epsilon}+\frac{1}{\tilde{k}}+\frac{1}{2}, \quad \frac{N}{2 \epsilon}+1<\frac{N}{2 \epsilon}+\frac{1}{2}+1-\frac{r}{2}
$$

$$
\frac{N}{2 \ell}+\frac{1}{\tilde{\ell}} \leq \frac{N}{2 \epsilon}+\frac{1}{\tilde{k}}+\frac{1}{2}, \quad \frac{N}{2 \ell}+\frac{1}{\tilde{\ell}}<\frac{N}{2 \epsilon}+\frac{1}{2}+1-\frac{r}{2}
$$

From Propositions 2.4 and 2.6, it follows that $p_{\xi}$ belongs to $L^{\tilde{k}}\left(T-\bar{t}, T ; W^{1, \epsilon}(\Omega)\right) \cap L^{2}\left(T-\bar{t}, T ; W^{r, \epsilon}(\Omega)\right)$. Let $\xi_{1}$ and $\xi_{2}$ belong to $L^{\tilde{k}}\left(T-\bar{t}, T ; W^{1, \epsilon}(\Omega)\right) \cap L^{2}\left(T-\bar{t}, T ; W^{r, \epsilon}(\Omega)\right)$. Still with Proposition 2.6, we have:

$\left\|p_{\xi_{1}}-p_{\xi_{2}}\right\|_{L^{\tilde{k}}\left(T-\bar{t}, T ; W^{1, \epsilon}(\Omega)\right)}+\left\|p_{\xi_{1}}-p_{\xi_{2}}\right\|_{L^{2}\left(T-\bar{t}, T ; W^{r, \epsilon}(\Omega)\right)} \leq C\|\vec{V}\|_{L^{\tilde{m}}\left(T-\bar{t}, T ;\left(L^{m}(\Omega)\right)^{N}\right)}\left\|\xi_{1}-\xi_{2}\right\|_{L^{\tilde{k}}\left(T-\bar{t}, T ; W^{1, \epsilon}(\Omega)\right)}$,

where $C$ is independent of $\bar{t}$. There exists $\bar{t}>0$ such that the mapping $\xi \mapsto p_{\xi}$ is a contraction in the Banach space $L^{\tilde{k}}\left(T-\bar{t}, T ; W^{1, \epsilon}(\Omega)\right) \cap L^{2}\left(T-\bar{t}, T ; W^{r, \epsilon}(\Omega)\right)$, and it admits a fixed point. As in the proof of Proposition 2.7, we can prove that equation (28) admits a unique solution in $L^{\tilde{k}}\left(0, T ; W^{1, \epsilon}(\Omega)\right) \cap L^{2}\left(0, T ; W^{r, \epsilon}(\Omega)\right)$. Since $2 \geq q^{\prime}$, and $r \epsilon>N-D \geq \sigma^{\prime}$, the trace of $p$ on $\left.\gamma \times\right] 0, T$ [ belongs to $L^{q^{\prime}}\left(0, T ; L^{\sigma^{\prime}}(\gamma)\right)$.

We have to study equation (26) in the case when $\vec{h}$ belongs to $L^{\frac{q}{\kappa-1}}\left(0, T ;\left(L^{\frac{d}{\kappa-1}}(\Omega)\right)^{N}\right)+L^{\frac{\delta_{2}}{\kappa-1}}\left(0, T ;\left(L^{\frac{\rho}{\kappa-1}}(\Omega)\right)^{N}\right.$ for all $1 \leq d<\frac{N-D}{N-D-1}$ and all $1 \leq \delta_{2}<2$, and $p_{T}$ belongs to $L^{\epsilon}(\Omega)$ for all $1 \leq \epsilon<\frac{N}{\left(N-\frac{D}{N-D}-\frac{2}{q^{\prime}}\right)(\theta-1)}$. In this case, due to Theorems 3.2 and 3.3, the trace of $p$ on $\gamma \times] 0, T$ [ belong to $L^{q^{\prime}}\left(0, T ; L^{\sigma^{\prime}}(\gamma)\right)$ if the following conditions are satisfied:

$$
\begin{gathered}
\frac{N-D}{(N-D-1)(\kappa-1)}>N-D>m^{\prime}, \\
\frac{\rho}{\kappa-1}>N-D>m^{\prime}, \\
\left(N-\frac{D}{N-D}-\frac{2}{q^{\prime}}\right)(\theta-1)<\frac{2}{q^{\prime}}+\frac{D}{N-D}, \quad\left(N-\frac{D}{N-D}-\frac{2}{q^{\prime}}\right)(\theta-1)<\frac{2}{\tilde{m}^{\prime}}+\frac{D}{N-D}, \\
\frac{q}{\kappa-1}>q^{\prime}, \quad \frac{2}{\kappa-1}>q^{\prime}, \quad \frac{2}{\kappa-1}>\tilde{m}^{\prime}, \quad \frac{q}{\kappa-1}>\tilde{m}^{\prime} .
\end{gathered}
$$

The condition $\frac{N-D}{(N-D-1)(\kappa-1)}>N-D$ is equivalent to $\kappa<1+\frac{1}{N-D-1}$. If $\kappa<1+\frac{1}{N-D-1}$, then $\kappa<2$, and the conditions $\frac{q}{\kappa-1}>q^{\prime}, \frac{2}{\kappa-1}>q^{\prime}, \frac{2}{\kappa-1}>\tilde{m}^{\prime}$, and $\frac{q}{\kappa-1}>\tilde{m}^{\prime}$ are automatically satisfied. Moreover, we have $N-D \geq \frac{N}{N-1}>m^{\prime}$, and $\rho=\frac{N}{N-\frac{D}{\sigma^{\prime}}-\frac{2}{q^{\prime}}} \geq \frac{N}{N-\frac{D}{N-D}-\frac{2}{q^{\prime}}}>\frac{N-D}{N-D-1}$. Thus $\frac{\rho}{\kappa-1}>N-D$ if $\frac{N-D}{(N-D-1)(\kappa-1)}>N-D$. 
We can summarize the above conditions in the assumptions stated below.

(A9) The exponent $\kappa$ satisfies

$$
1<\kappa<1+\frac{1}{N-D-1}
$$

(A10) The exponent $\theta$ satisfies

$$
\begin{aligned}
& (\theta-1)\left(N-\frac{D}{N-D}-\frac{2}{q^{\prime}}\right)<\frac{2}{q^{\prime}}+\frac{D}{N-D}, \\
& (\theta-1)\left(N-\frac{D}{N-D}-\frac{2}{q^{\prime}}\right)<\frac{2}{\tilde{m}^{\prime}}+\frac{D}{N-D} .
\end{aligned}
$$

These assumptions are satisfied in the following cases. When $N=3, D=1, \tilde{m}=8, m=4, q=\sigma=2$, we can set $\rho=2$, and (A9, A10) are satisfied for all $1<\kappa<2$, and all $1<\theta<2$. In the case where $N=3, D=1, \tilde{m}=8, m=4, q=\sigma=\infty$, we can set $\rho=\infty$, and the previous assumptions are satisfied for all $1<\kappa<2$, and all $1<\theta<\frac{11}{2}$.

Theorem 3.4. Set $\vec{h}=\kappa C_{Q}\left|\nabla y_{u}-V_{d}\right|^{\kappa-2}\left(\nabla y_{u}-V_{d}\right)$, and $p_{T}=\theta C_{\Omega}\left|y_{u}(T)-y_{d}\right|^{\theta-2}\left(y_{u}(T)-y_{d}\right)$, where $y_{u}$ is the solution of (1) corresponding to $u \in L^{q}\left(0, T ; L^{\sigma}(\gamma)\right)$. Let $p$ be the solution to the equation

$$
-\frac{\partial p}{\partial t}+A p-\vec{V} \cdot \nabla p=-\operatorname{div} \vec{h} \text { in } Q, \quad \frac{\partial p}{\partial n_{A}}=\vec{h} \cdot \vec{n} \text { on } \Sigma, \quad p(T)=p_{T} \text { in } \Omega .
$$

Then the trace of $p$ on $\gamma \times] 0, T\left[\right.$ belongs to $L^{q^{\prime}}\left(0, T ; L^{\sigma^{\prime}}(\gamma)\right)$.

Proof. From assumption (33), it follows that $\vec{h} \in L^{\tilde{\eta}_{1}}\left(0, T ; L^{\eta_{1}}(\Omega)\right)+L^{\tilde{\eta}_{2}}\left(0, T ; L^{\eta_{2}}(\Omega)\right)$, for some $\left(\tilde{\eta}_{1}, \eta_{1}\right),\left(\tilde{\eta}_{2}, \eta_{2}\right)$ satisfying $\tilde{\eta}_{i}>\tilde{m}^{\prime}, \tilde{\eta}_{i} \geq q^{\prime}, \eta_{i}>N-D>m^{\prime}$ for $i=1,2$. From assumptions $(34,35)$, it follows that $p_{T} \in L^{\epsilon}(\Omega)$ for some $\epsilon$ satisfying $\frac{N}{2 \epsilon}<\frac{1}{\tilde{m}^{\prime}}+\frac{D}{2(N-D)}$ and $\frac{N}{2 \epsilon}<\frac{1}{q^{\prime}}+\frac{D}{2(N-D)}$. Thus, the theorem is a direct consequence of Theorems 3.2 and 3.3 .

\subsection{Optimality conditions for $\left(\boldsymbol{P}_{\mathbf{1}}\right)$}

Proposition 3.1. Let $u \in L^{q}\left(0, T ; L^{\sigma}(\gamma)\right)$ and $y_{u}$ be the solution of (1) corresponding to $u$. Let $p$ be the solution to the adjoint equation (36). Let $z$ be the solution to the equation

$$
\frac{\partial z}{\partial t}+A z+\vec{V} \cdot \nabla z=0 \text { in } Q, \quad \frac{\partial z}{\partial n_{A}}=u \delta_{\gamma} \text { on } \Sigma, \quad z(0)=0 \text { in } \Omega .
$$

Then

$$
\int_{0}^{T} \int_{\gamma} p u \mathrm{~d} \zeta \mathrm{d} t=\int_{Q} \nabla z \cdot \vec{h} \mathrm{~d} x \mathrm{~d} t+\int_{\Omega} p_{T}(x) z(x, T) \mathrm{d} x .
$$

Proof. Observe that $z(T)$ belongs to $L^{r}(\Omega)$ for all $1 \leq r<\frac{N}{N-\frac{D}{N-D}-\frac{2}{q^{\prime}}}$. With condition (34), we can find $\epsilon$ satisfying $\epsilon<\frac{N}{\left(N-\frac{D}{N-D}-\frac{2}{q^{\prime}}\right)(\theta-1)}$, such that $z(T)$ belongs to $L^{\epsilon^{\prime}}(\Omega)$. Thus $p_{T} z(T)$ belongs to $L^{1}(\Omega)$. Due to 
Proposition 2.7, $z$ belongs to $L^{q}\left(0, T ; W^{1, d}(\Omega)\right)$ for all $d<\frac{N-D}{N-D-1}$. Set $\vec{h}=\overrightarrow{h^{1}}+\overrightarrow{h^{2}}$, where $\overrightarrow{h^{1}}$ (respectively $\left.\overrightarrow{h^{2}}\right)$ belongs to $L^{\frac{q}{\kappa-1}}\left(0, T ;\left(L^{\frac{d}{\kappa-1}}(\Omega)\right)^{N}\right)$ for all $d<\frac{N-D}{N-D-1}\left(\operatorname{respectively~} L^{\frac{\delta_{2}}{\kappa-1}}\left(0, T ;\left(L^{\frac{\rho}{\kappa-1}}(\Omega)\right)^{N}\right)\right.$ for all $\left.\delta_{2}<2\right)$. Let $\left(\overrightarrow{h_{k}^{1}}\right)_{k}$ (respectively $\left.\left(\overrightarrow{h_{k}^{2}}\right)_{k}\right)$ be a sequence of functions in $(\mathcal{D}(Q))^{N}$ converging to $\overrightarrow{h^{1}}$ (respectively $\left.\overrightarrow{h^{2}}\right)$ in $L^{\frac{q}{\kappa-1}}\left(0, T ;\left(L^{\frac{d}{\kappa-1}}(\Omega)\right)^{N}\right)$ for all $d<\frac{N-D}{N-D-1}\left(\right.$ respectively $L^{\frac{\delta_{2}}{\kappa-1}}\left(0, T ;\left(L^{\frac{\rho}{\kappa-1}}(\Omega)\right)^{N}\right)$ for all $\left.\delta_{2}<2\right)$. Due to assumption (A9), there exists $\delta_{2}<2$ and $d<\frac{N-D}{N-D-1}$ obeying $\frac{\kappa-1}{\delta_{2}}+\frac{1}{q} \leq 1, \frac{\kappa-1}{\rho}+\frac{1}{d} \leq 1$. Then $\nabla z \cdot\left(\overrightarrow{h_{k}^{1}}+\overrightarrow{h_{k}^{2}}\right)$ belongs to $L^{1}(Q)$, and converges, when $k$ tends to infinity, to $\nabla z \cdot \vec{h}$ in $L^{1}(Q)$. Let $\left(p_{T}^{k}\right)_{k}$ be a sequence of regular functions converging to $p_{T}$ in $L^{\epsilon}(\Omega)$ for all $1 \leq \epsilon<\frac{N}{\left(N-\frac{D}{N-D}-\frac{2}{q^{\prime}}\right)(\theta-1)}$, and $p_{k}$ be the solution to the equation:

$$
-\frac{\partial p_{k}}{\partial t}+A p_{k}-\vec{V} \cdot \nabla p=-\operatorname{div}\left(\overrightarrow{h_{k}^{1}}+\overrightarrow{h_{k}^{2}}\right) \text { in } Q, \quad \frac{\partial p_{k}}{\partial n_{A}}=0 \text { on } \Sigma, \quad p_{k}(T)=p_{T}^{k} \text { in } \Omega
$$

Then we have

$$
\int_{0}^{T} \int_{\gamma} p_{k}(\zeta, t) u(\zeta, t) \mathrm{d} \zeta \mathrm{d} t=\int_{Q} \nabla z \cdot\left(\overrightarrow{h_{k}^{1}}+\overrightarrow{h_{k}^{2}}\right) \mathrm{d} x \mathrm{~d} t+\int_{\Omega} p_{T}^{k}(x) z(x, T) \mathrm{d} x .
$$

By passing to the limit when $k$ tends to $\infty$, we obtain:

$$
\int_{0}^{T} \int_{\gamma} p(\zeta, t) u(\zeta, t) \mathrm{d} \zeta \mathrm{d} t=\int_{Q} \nabla z \cdot\left(\overrightarrow{h^{1}}+\overrightarrow{h^{2}}\right) \mathrm{d} x \mathrm{~d} t+\int_{\Omega} p_{T}(x) z(x, T) \mathrm{d} x
$$

Theorem 3.5. If $u$ is a solution of $\left(P_{1}\right)$, then

$$
\int_{0}^{T} \int_{\gamma} p(v-u) \mathrm{d} \zeta \mathrm{d} t+q C_{\gamma} \int_{0}^{T}\left(\int_{\gamma}|u|^{\sigma} \mathrm{d} \zeta\right)^{\frac{q}{\sigma}-1}\left(\int_{\gamma}|u|^{\sigma-2} u(v-u) \mathrm{d} \zeta\right) \mathrm{d} t \geq 0
$$

for every $v \in K_{U}$, where $p$ is the solution to

$$
\begin{gathered}
-\frac{\partial p}{\partial t}+A p-\vec{V} \cdot \nabla p=-\kappa C_{Q} \operatorname{div}\left(\left|\nabla y_{u}-V_{d}\right|^{\kappa-2}\left(\nabla y_{u}-V_{d}\right)\right) \text { in } Q \\
\frac{\partial p}{\partial n_{A}}=\kappa C_{Q}\left(\left|\nabla y_{u}-V_{d}\right|^{\kappa-2}\left(\nabla y_{u}-V_{d}\right)\right) \cdot \vec{n} \text { on } \Sigma, \quad p(T)=\theta C_{\Omega}\left|y_{u}(T)-y_{d}\right|^{\theta-2}\left(y_{u}(T)-y_{d}\right) \text { in } \Omega
\end{gathered}
$$

where $y_{u}$ is the solution to equation (1) corresponding to $u$.

Proof. Let $v$ be in $K_{U}, \lambda>0$, and denote by $y_{\lambda}$ the solution of (1) corresponding to $u+\lambda(v-u)$. Due to (34), applying Proposition 2.7, $y_{\lambda}$ and $y_{u}$ belong to $C\left([0, T] ; L_{w}^{\theta}(\Omega)\right)$.

1 - We set $w_{\lambda}=y_{\lambda}-y_{u}$, then $w_{\lambda}$ is the solution to the equation:

$$
\frac{\partial w}{\partial t}+A w+\vec{V} \cdot \nabla w=0 \text { in } Q, \quad \frac{\partial w}{\partial n_{A}}=\lambda(v-u) \delta_{\gamma} \text { on } \Sigma, \quad w(0)=0 \text { in } \Omega .
$$

With Proposition 2.7 and condition $(34)$, for all $(\delta, d)$ satisfying $(17)$, we have the estimate:

$$
\left\|y_{\lambda}-y_{u}\right\|_{L^{\delta}\left(0, T ; W^{1, d}(\Omega)\right)}+\left\|y_{\lambda}(T)-y_{u}(T)\right\|_{L^{\theta}(\Omega)} \leq C \lambda\|v-u\|_{L^{q}\left(0, T ; L^{\sigma}(\gamma)\right)} .
$$


2 - Let us set $z=\left(y_{\lambda}-y_{u}\right) / \lambda$. Observe that $z$ is independent of $\lambda$. Due to Proposition $2.7, z$ belongs to $L^{\delta}\left(0, T ; W^{1, d}(\Omega)\right)$ for all $(\delta, d)$ satisfying $(17)$, and $z(T)$ belongs to $L^{r}(\Omega)$, for all $1 \leq r<\frac{N}{\left(N-\frac{D}{N-D}-\frac{2}{q^{\prime}}\right)}$.

3 - We want to calculate the gradient of the functional $I$. If we set $G(u)=\int_{\Omega}\left|y_{u}(T)-y_{d}\right|^{\theta} \mathrm{d} x+\int_{Q}\left|\nabla y_{u}-V_{d}\right|^{\kappa} \mathrm{d} x \mathrm{~d} t$, from the convexity of the mapping $y \mapsto \int_{\Omega}\left|y(T)-y_{d}\right|^{\theta} \mathrm{d} x+\int_{Q}\left|\nabla y-V_{d}\right|^{\kappa} \mathrm{d} x \mathrm{~d} t$, it follows that:

$$
\begin{gathered}
\quad \theta C_{\Omega} \int_{\Omega}\left|y_{u}(T)-y_{d}\right|^{\theta-2}\left(y_{u}(T)-y_{d}\right) z(T) \mathrm{d} x+\kappa C_{Q} \int_{Q}\left|\nabla y_{u}-V_{d}\right|^{\kappa-2}\left(\nabla y_{u}-V_{d}\right) \nabla z \mathrm{~d} x \\
\leq \frac{1}{\lambda}(G(u+\lambda(v-u))-G(u)) \\
\leq \theta C_{\Omega} \int_{\Omega}\left|y_{\lambda}(T)-y_{d}\right|^{\theta-2}\left(y_{\lambda}(T)-y_{d}\right) z(T) \mathrm{d} x+\kappa C_{Q} \int_{Q}\left|\nabla y_{\lambda}-V_{d}\right|^{\kappa-2}\left(\nabla y_{\lambda}-V_{d}\right) \nabla z \mathrm{~d} x .
\end{gathered}
$$

We set $p_{T}=\theta C_{\Omega}\left|y_{u}(T)-y_{d}\right|^{\theta-2}\left(y_{u}(T)-y_{d}\right)$, and $p_{T}^{\lambda}=\theta C_{\Omega}\left|y_{\lambda}(T)-y_{d}\right|^{\theta-2}\left(y_{\lambda}(T)-y_{d}\right)$. From (43), it follows that $p_{T}$ and $p_{T}^{\lambda}$ belong to $L^{\theta^{\prime}}(\Omega)$, and that $\int_{\Omega} p_{T}^{\lambda} z(T) \mathrm{d} x \longmapsto \int_{\Omega} p_{T} z(T) \mathrm{d} x$ as $\lambda$ tends to zero.

We set $\vec{h}=\kappa C_{Q}\left|\nabla y_{u}-V_{d}\right|^{\kappa-2}\left(\nabla y_{u}-V_{d}\right)$, and $\vec{h}_{\lambda}=\kappa C_{Q}\left|\nabla y_{\lambda}-V_{d}\right|^{\kappa-2}\left(\nabla y_{\lambda}-V_{d}\right)$. Since $q \geq 2$ we have $\kappa \leq q$ and $\frac{\kappa-1}{2}+\frac{1}{q}<1$. Due to assumptions (33), we can find $d$ such that $\kappa \leq d<\frac{N-D}{N-D-1}, \frac{\kappa-1}{\rho}+\frac{N-D-1}{N-D}<\frac{\kappa-1}{\rho}+\frac{1}{d} \leq 1$. Thus we can choose $1<\delta_{2}<2$ such that $\frac{\kappa-1}{2}+\frac{1}{q}<\frac{\kappa-1}{\delta_{2}}+\frac{1}{q} \leq 1$. We can verify that $\vec{h}_{\lambda} \cdot \nabla z$ belongs to $L^{1}(Q)$, and $\int_{Q} \vec{h}_{\lambda} \cdot \nabla z \mathrm{~d} x \mathrm{~d} t \longmapsto \int_{Q} \vec{h} \cdot \nabla z \mathrm{~d} x \mathrm{~d} t$ as $\lambda$ tends to zero. Therefore, if we set $F(u)=I\left(y_{u}, u\right)$, thanks to the above calculations, we obtain:

$$
\begin{aligned}
F^{\prime}(u)(v-u)= & \theta C_{\Omega} \int_{\Omega}\left|y_{u}(T)-y_{d}\right|^{\theta-2}\left(y_{u}(T)-y_{d}\right) z(T) \mathrm{d} x+\kappa C_{Q} \int_{Q}\left|\nabla y_{u}-V_{d}\right|^{\kappa-2}\left(\nabla y_{u}-V_{d}\right) \cdot \nabla z \mathrm{~d} x \\
& +q C_{\gamma} \int_{0}^{T}\left(\int_{\gamma}|u|^{\sigma} \mathrm{d} \zeta\right)^{\frac{q}{\sigma}-1}\left(\int_{\gamma}|u|^{\sigma-2} u(v-u) \mathrm{d} \zeta\right) \mathrm{d} t
\end{aligned}
$$

Finally we can use the Green formula (38) to complete the proof.

\section{Control problem $\left(\mathrm{P}_{2}\right)$}

In this section we study the control problem $\left(P_{2}\right)$. In this case $\delta_{\gamma}$ is replaced by $\delta_{x_{0}}$, which corresponds to $D=0$. We first prove the existence of an optimal pair $\left(u, x_{0}\right)$, and next establish optimality conditions. For this, we make the following assumptions.

(A6') The function $y_{d}$ belongs to $L^{\frac{N}{N-2}}(\Omega), K_{U}$ is a closed convex bounded subset of $L^{\infty}(0, T)$, and $K_{\bar{\Omega}}$ is a closed convex subset of $\bar{\Omega}$. (Observe that $q=\sigma=\infty$.)

(A7') The exponent $\theta$ satisfies the condition

$$
\theta<\frac{N-1}{N-2}
$$

\subsection{Existence of solutions to $\left(\mathbf{P}_{\mathbf{2}}\right)$}

Theorem 4.1. Assume that conditions (A1-A5) and (A6') are fulfilled. Suppose that $\theta<\frac{N}{N-2}$. Then the optimal control problem $\left(P_{2}\right)$ admits solutions.

Proof. Let $\left(u_{n}, x_{n}\right)_{n}$ be a minimizing sequence in $K_{U} \times K_{\bar{\Omega}}$. Then $\left(u_{n}, x_{n}\right)_{n}$ is bounded in $L^{\infty}(0, T) \times R^{N}$. We can suppose that $\left(u_{n}\right)_{n}$ converges to some $u$ weakly-star in $L^{\infty}(0, T)$, and $\left(x_{n}\right)_{n}$ converges in $R^{N}$ to some $x_{0} \in K_{\bar{\Omega}}$. Since $K_{U}$ is convex and closed in $L^{\infty}(0, T), u$ belongs to $K_{U}$. Let $y_{n}$ be the solution to (1) corresponding to $\left(u_{n}, x_{n}\right)$, and $y_{u, x_{0}}$ be the solution of $(1)$ corresponding to $\left(u, x_{0}\right)$. As in the proof of Theorem 3.1, 
we can prove that $\left(y_{n}\right)_{n}$ converges to $y_{u, x_{0}}$ for the weak topology of $L^{\delta}\left(0, T ; W^{1, d}(\Omega)\right)+L^{\delta_{2}}\left(0, T ; W^{1, d_{2}}(\Omega)\right)$ for all $(\delta, d)$ satisfying $(17)$ with $D=0$, and all $\left(\delta_{2}, d_{2}\right)$ satisfying $(24)$ with $D=0$. Moreover, we can prove that $\left(y_{n}(T)\right)_{n}$ converges to $y_{u, x_{0}}(T)$ for the weak topology of $L^{\theta}(\Omega)$. By classical arguments, we can next prove that $\left(u, x_{0}\right)$ is a solution of $\left(P_{2}\right)$.

\subsection{Regularity results of the adjoint state}

We consider the following terminal boundary value problem

$$
-\frac{\partial p}{\partial t}+A p-\vec{V} \cdot \nabla p=0 \text { in } Q, \quad \frac{\partial p}{\partial n_{A}}=0 \text { on } \Sigma, \quad p(T)=p_{T} \text { in } \Omega,
$$

where $p_{T}$ belongs to $\left.L^{\epsilon}(\Omega)\right)$ for all $1 \leq \epsilon<\frac{N}{(N-2)(\theta-1)}$. If $\bar{y}$ is the solution of (1) corresponding to $u$ and $x_{0}$, if we set $p_{T}=\theta\left|\bar{y}(T)-y_{d}\right|^{\theta-2}\left(\bar{y}(T)-y_{d}\right)$, then equation (45) corresponds to the adjoint equation for $J$ associated with $\left(x_{0}, u\right)$.

Theorem 4.2. The solution $p$ to equation (45) belongs to $L^{1}\left(0, T ; C^{1}(\bar{\Omega})\right)$. Moreover, for all $N<\epsilon<$ $\frac{N}{(N-2)(\theta-1)}$, there exists $0<\nu<1$, such that the mapping that associates $p$ with $p_{T}$ is continuous from $L^{\epsilon}(\Omega)$ into $L^{1}\left(0, T ; C^{1, \nu}(\bar{\Omega})\right)$.

Proof. 1 - Due to condition (44) on $\theta$, we can find $\epsilon$ such that $N<\epsilon<\frac{N}{(N-2)(\theta-1)}$. Then $\epsilon>m^{\prime}$. We choose $(\alpha, \xi)$ satisfying

$$
\alpha>m>N, \quad \frac{1}{2}+\frac{N}{2 \alpha}<\frac{\xi}{2}<\inf \left\{1+\frac{N}{2 \alpha}-\frac{N}{2 \epsilon}, \frac{3}{2}-\frac{1}{\tilde{m}}-\frac{N}{2 m}+\frac{N}{2 \alpha}-\frac{N}{2 \epsilon}, 1-\frac{N}{2 m}+\frac{N}{2 \alpha}\right\} .
$$

Since $\frac{N}{2 \alpha}-\frac{N}{2 m}<0$, then $\frac{\xi}{2}<1<1+\frac{1}{\tilde{m}^{\prime}}$. Thus there exists $\tilde{\alpha}$ such that

$$
\begin{gathered}
\sup \left\{\frac{\xi}{2}-\frac{1}{\tilde{m}^{\prime}}, \frac{1}{2}\right\}<\frac{1}{\tilde{\alpha}}<1, \\
-\frac{1}{2}+\frac{\xi}{2}+\frac{1}{\tilde{m}}+\frac{N}{2 m}-\frac{N}{2 \alpha}+\frac{N}{2 \epsilon}<\frac{1}{\tilde{\alpha}}<1 .
\end{gathered}
$$

With (48), we have $\frac{1}{2}-\frac{1}{\tilde{m}^{\prime}}+\frac{N}{2 \epsilon}<1-\frac{\xi}{2}-\frac{1}{\tilde{m}}-\frac{N}{2 m}+\frac{1}{\tilde{\alpha}}+\frac{N}{2 \alpha}$, and $0<\frac{1}{2}+\frac{N}{2 \epsilon}<1-\frac{\xi}{2}-\frac{1}{\tilde{m}}-\frac{N}{2 m}+\frac{1}{\tilde{\alpha}}+\frac{N}{2 \alpha}$. From (46), we deduce that $1-\frac{\xi}{2}-\frac{N}{2 m}+\frac{N}{2 \alpha}>0$ and $1-\frac{\xi}{2}-\frac{N}{2 m}+\frac{N}{2 \alpha}>\frac{1}{2}-\frac{1}{\tilde{m}^{\prime}}+\frac{N}{2 \epsilon}$. Thus there exists $k$ satisfying

$$
\sup \left\{\frac{1}{2}-\frac{1}{\tilde{m}^{\prime}}+\frac{N}{2 \epsilon}, 0\right\}<\frac{N}{2 k}<\inf \left\{\frac{N}{2 \epsilon}, 1-\frac{\xi}{2}-\frac{1}{\tilde{m}}-\frac{N}{2 m}+\frac{1}{\tilde{\alpha}}+\frac{N}{2 \alpha}, 1-\frac{\xi}{2}-\frac{N}{2 m}+\frac{N}{2 \alpha}\right\} .
$$

With (49), we obtain $\frac{1}{2}+\frac{N}{2 \epsilon}-\frac{N}{2 k}<\frac{1}{\tilde{m}^{\prime}}$, and $-\frac{1}{\tilde{m}}+\frac{1}{\tilde{\alpha}}<1-\frac{\xi}{2}-\frac{1}{\tilde{m}}-\frac{N}{2 m}+\frac{1}{\tilde{\alpha}}+\frac{N}{2 \alpha}-\frac{N}{2 k}$. From (48), we deduce that $\frac{1}{2}+\frac{N}{2 \epsilon}-\frac{N}{2 k}<1-\frac{\xi}{2}-\frac{1}{\tilde{m}}-\frac{N}{2 m}+\frac{1}{\tilde{\alpha}}+\frac{N}{2 \alpha}-\frac{N}{2 k}$. Thus we can choose $\tilde{k}$ such that

$$
\sup \left\{-\frac{1}{\tilde{m}}+\frac{1}{\tilde{\alpha}}, \frac{1}{2}+\frac{N}{2 \epsilon}-\frac{N}{2 k}\right\}<\frac{1}{\tilde{k}}<\inf \left\{\frac{1}{\tilde{m}^{\prime}}, 1-\frac{\xi}{2}-\frac{1}{\tilde{m}}-\frac{N}{2 m}+\frac{1}{\tilde{\alpha}}+\frac{N}{2 \alpha}-\frac{N}{2 k}\right\} .
$$

With (49), we have $k>\epsilon>m^{\prime}$. Due to (50), the pair $(\tilde{k}, k)$ obeys (29). From Theorem 3.3, we deduce that $p$ belongs to $L^{\tilde{k}}\left(0, T ; W^{1, k}(\Omega)\right)$. 
2 - We set $\frac{1}{\ell}=\frac{1}{\tilde{m}}+\frac{1}{k}$ and $\frac{1}{\ell}=\frac{1}{m}+\frac{1}{k}$, then $\tilde{\ell}>1$. From $\frac{1}{m}+\frac{1}{k}<\frac{1}{m}+\frac{1}{\epsilon}<\frac{1}{m}+\frac{1}{m^{\prime}}=1$, it follows that $\ell>1$ and $\vec{V} \cdot \nabla p \in L^{\tilde{\ell}}\left(0, T ; L^{\ell}(\Omega)\right)$. Let $\pi$ be the solution to the equation:

$$
-\frac{\partial \pi}{\partial t}+A \pi=\vec{V} \cdot \nabla p \text { in } Q, \quad \frac{\partial \pi}{\partial n_{A}}=0 \text { on } \Sigma, \quad \pi(T)=0 \text { in } \Omega .
$$

From the choice of $\tilde{k}$ and $k$, with $\frac{1}{\alpha}<\frac{1}{m}<\frac{1}{m}+\frac{1}{k}$, we obtain $\tilde{\alpha} \geq \tilde{\ell}, \alpha \geq \ell, \frac{1}{\tilde{\ell}}+\frac{N}{2 \ell}<\frac{1}{\tilde{\alpha}}+\frac{N}{2 \alpha}+1-\frac{\xi}{2}$. Due to Proposition 2.6, we deduce that $\pi$ belongs to $L^{\tilde{\alpha}}\left(0, T ; W^{\xi, \alpha}(\Omega)\right)$.

3 - Let $\pi_{2}$ be the solution to the equation:

$$
-\frac{\partial \pi_{2}}{\partial t}+A \pi_{2}=0 \text { in } Q, \quad \frac{\partial \pi_{2}}{\partial n_{A}}=0 \text { on } \Sigma, \quad \pi_{2}(T)=p_{T} \text { in } \Omega .
$$

Let $\left(\alpha_{2}, \xi_{2}\right)$ be a pair satisfying

$$
\alpha_{2} \geq \epsilon, \quad 1+\frac{N}{2 \epsilon}<\frac{N}{2 \alpha_{2}}+2-\frac{\xi_{2}}{2}<\frac{3}{2} .
$$

From Proposition 2.4, we deduce that $\pi_{2}$ belongs to $L^{1}\left(0, T ; W^{\xi_{2}, \alpha_{2}}(\Omega)\right)$, which is included in $L^{1}\left(0, T ; C^{1}(\bar{\Omega})\right)$. 4 - Let $(\alpha, \xi)$ obey $(46)$, and $\left(\alpha_{2}, \xi_{2}\right)$ obey (51). Then there exists $\nu$ such that

$$
1>\nu>0, \quad \xi>\nu+1+\frac{N}{\alpha}, \quad \xi_{2}>\nu+1+\frac{N}{\alpha_{2}} .
$$

We have $p=\pi+\pi_{2} \in L^{1}\left(0, T ; W^{\xi, \alpha}(\Omega)\right)+L^{1}\left(0, T ; W^{\xi_{2}, \alpha_{2}}(\Omega)\right)$, and $L^{1}\left(0, T ; W^{\xi, \alpha}(\Omega)\right)+L^{1}\left(0, T ; W^{\xi_{2}, \alpha_{2}}(\Omega)\right)$ is included in $L^{1}\left(0, T ; C^{1, \nu}(\bar{\Omega})\right)$. The estimate of $p$ in $L^{1}\left(0, T ; C^{1, \nu}(\bar{\Omega})\right)$ in function of $\left\|p_{T}\right\|_{L^{\epsilon}(\Omega)}$ may be deduced from the analysis of Step 1 and Step 2.

\subsection{Optimality conditions}

Lemma 4.1. Let $p$ be the solution of (45), where $p_{T}$ belongs to $\left.L^{\epsilon}(\Omega)\right)$ for all $1 \leq \epsilon<\frac{N}{(N-2)(\theta-1)}$. Let $\left(p_{T}^{\lambda}\right)_{\lambda}$ be a sequence of functions converging to $p_{T}$ for the weak topology of the space $L^{\epsilon}(\Omega)$ for all $\epsilon<\frac{N}{(N-2)(\theta-1)}$. Let $p_{\lambda}$ be the solution to the equation:

$$
-\frac{\partial p}{\partial t}+A p-\vec{V} \cdot \nabla p=0 \text { in } Q, \quad \frac{\partial p}{\partial n_{A}}=0 \text { on } \Sigma, \quad p(T)=p_{T}^{\lambda} \text { in } \Omega .
$$

Then the sequence $\left(p_{\lambda}\right)_{\lambda}$ converges to $p$ in $L^{1}\left(0, T ; C^{1}(\bar{\Omega})\right)$.

Proof. From Theorem 4.2, we know that the sequence $\left(p_{\lambda}\right)_{\lambda}$ is bounded in $L^{1}\left(0, T ; C^{1, \nu}(\bar{\Omega})\right)$ for some $0<$ $\nu<1$. The identity mapping from $C^{1, \nu}(\bar{\Omega})$ into $C^{1}(\bar{\Omega})$ is compact. The sequence $\left(\frac{d p_{\lambda}}{d t}\right)_{\lambda}$ is bounded in $L^{1}\left(0, T ;\left(W^{1, \beta}(\Omega)\right)^{\prime}\right)$ for some $\beta$ big enough. From a compactness result ([25], Cor. 5), we deduce that the identity mapping is compact from $L^{1}\left(0, T ; C^{1, \nu}(\bar{\Omega})\right) \cap W^{1,1}\left(0, T ;\left(W^{1, \beta}(\Omega)\right)^{\prime}\right)$ into $L^{1}\left(0, T ; C^{1}(\bar{\Omega})\right)$. Therefore the sequence $\left(p_{\lambda}\right)_{\lambda}$ converges to $p$ in $L^{1}\left(0, T ; C^{1}(\bar{\Omega})\right)$.

Lemma 4.2. Let $p$ and $p_{\lambda}$ be defined as in Lemma 4.1. Then we have

$$
\lim _{\lambda \rightarrow 0} \frac{1}{\lambda} \int_{0}^{T}\left(p_{\lambda}\left(x_{0}+\lambda\left(x_{1}-x_{0}\right)\right)-p_{\lambda}\left(x_{0}\right)\right) u \mathrm{~d} t=\int_{0}^{T} \nabla p\left(x_{0}\right) \cdot\left(x_{1}-x_{0}\right) u \mathrm{~d} t .
$$


Proof. We can write:

$$
\begin{aligned}
& \frac{1}{\lambda} \int_{0}^{T}\left(p_{\lambda}\left(x_{0}+\lambda\left(x_{1}-x_{0}\right)\right)-p_{\lambda}\left(x_{0}\right)\right) u \mathrm{~d} t=\int_{0}^{T} \int_{0}^{1} \nabla p_{\lambda}\left(x_{0}+\vartheta \lambda\left(x_{1}-x_{0}\right)\right) \cdot\left(x_{1}-x_{0}\right) u \mathrm{~d} \vartheta \mathrm{d} t \\
& =\int_{0}^{T} \int_{0}^{1}\left(\nabla p_{\lambda}\left(x_{0}+\vartheta \lambda\left(x_{1}-x_{0}\right)\right)-\nabla p_{\lambda}\left(x_{0}\right)\right) \cdot\left(x_{1}-x_{0}\right) u \mathrm{~d} \vartheta \mathrm{d} t+\int_{0}^{T} \nabla p_{\lambda}\left(x_{0}\right) \cdot\left(x_{1}-x_{0}\right) u \mathrm{~d} t .
\end{aligned}
$$

Due to Lemma 4.1 , the sequence $\left(p_{\lambda}\right)_{\lambda}$ converges to $p$ in $L^{1}\left(0, T ; C^{1}(\bar{\Omega})\right)$. Thus we have:

$$
\int_{0}^{T} \nabla p_{\lambda}\left(x_{0}\right) \cdot\left(x_{1}-x_{0}\right) u \mathrm{~d} t \longrightarrow \int_{0}^{T} \nabla p\left(x_{0}\right) \cdot\left(x_{1}-x_{0}\right) u \mathrm{~d} t \quad \text { as } \lambda \rightarrow 0 .
$$

Due to Theorem $4.2,\left(p_{\lambda}\right)_{\lambda}$ is bounded in $L^{1}\left(0, T ; C^{1, \nu}(\bar{\Omega})\right)$ for some $0<\nu<1$. Therefore we have

$$
\begin{gathered}
\left|\int_{0}^{T} \int_{0}^{1}\left(\nabla p_{\lambda}\left(x_{0}+\vartheta \lambda\left(x_{1}-x_{0}\right)\right)-\nabla p_{\lambda}\left(x_{0}\right)\right) \cdot\left(x_{1}-x_{0}\right) u \mathrm{~d} \vartheta \mathrm{d} t\right| \\
\leq \int_{0}^{T} \int_{0}^{1} \frac{\left|\left(\nabla p_{\lambda}\left(x_{0}+\vartheta \lambda\left(x_{1}-x_{0}\right)\right)-\nabla p_{\lambda}\left(x_{0}\right)\right) \cdot\left(x_{1}-x_{0}\right)\right|}{\left|x_{0}+\vartheta \lambda\left(x_{1}-x_{0}\right)-x_{0}\right|^{\nu}} \mathrm{d} \vartheta\left|\lambda\left(x_{1}-x_{0}\right)\right|^{\nu}|u| \mathrm{d} t \\
\leq C\|u\|_{L^{\infty}(0, T)}|| p_{\lambda} \|_{L^{1}\left(0, T ; C^{1, \nu}(\bar{\Omega})\right)}\left|\lambda\left(x_{1}-x_{0}\right)\right|^{\nu} \longrightarrow 0 \quad \text { as } \lambda \rightarrow 0 .
\end{gathered}
$$

The proof is complete.

Theorem 4.3. If $\left(u, x_{0}\right)$ is a solution of $\left(P_{2}\right)$, then

$$
\int_{0}^{T} p\left(x_{0}\right)(v-u) \mathrm{d} t \geq 0 \text { for all } v \in K_{U}, \text { and } \int_{0}^{T} \nabla p\left(x_{0}\right) \cdot\left(x_{1}-x_{0}\right) u \mathrm{~d} t \geq 0, \text { for all } x_{1} \in K_{\bar{\Omega}},
$$

where $p$ is the solution to the equation

$$
-\frac{\partial p}{\partial t}+A p-\vec{V} \cdot \nabla p=0 \text { in } Q, \quad \frac{\partial p}{\partial n_{A}}=0 \text { on } \Sigma, \quad p(T)=\theta\left|\bar{y}(T)-y_{d}\right|^{\theta-2}\left(\bar{y}(T)-y_{d}\right) \text { in } \Omega
$$

and where $\bar{y}$ is the solution of (1) corresponding to $u$ and $x_{0}$.

Proof. We only prove the optimality condition for $x_{0}$. For $\lambda>0$, we denote by $y_{\lambda}$ the solution of (1) corresponding to $u$ and $x_{0}+\lambda\left(x_{1}-x_{0}\right)$. Set $F(x)=J\left(y_{u, x}, u, x\right)$. We set $z_{\lambda}=\left(y_{\lambda}-y\right) / \lambda$, then $z_{\lambda}$ is the solution to the equation:

$$
\frac{\partial z}{\partial t}+A z+\vec{V} \cdot \nabla z=0 \text { in } Q, \quad \frac{\partial z}{\partial n_{A}}=u(t)\left(\delta_{x_{0}+\lambda\left(x_{1}-x_{0}\right)}-\delta_{x_{0}}\right) / \lambda \text { on } \Sigma, \quad z(0)=0 \text { in } \Omega .
$$

Under conditions (44) on $\theta$, and applying Proposition 2.7, we deduce that $y_{\lambda}$ and $z_{\lambda}$ belong to $C\left([0, T] ; L_{w}^{\theta}(\Omega)\right)$. Then $\left|y_{\lambda}(T)-y_{d}\right|^{\theta-2}\left(y_{\lambda}(T)-y_{d}\right) z_{\lambda}(T)$ belongs to $L^{1}(\Omega)$. From the convexity of the mapping $y \mapsto \int_{\Omega} \mid y(T)-$ $\left.y_{d}\right|^{\theta} \mathrm{d} x$, it follows that

$$
0 \leq \frac{F\left(x_{0}+\lambda\left(x_{1}-x_{0}\right)\right)-F\left(x_{0}\right)}{\lambda} \leq \theta \int_{\Omega}\left|y_{\lambda}(T)-y_{d}\right|^{\theta-2}\left(y_{\lambda}(T)-y_{d}\right) z_{\lambda}(T) \mathrm{d} x .
$$

Using the Green formula of Proposition 3.1 with $\vec{h}=0$, we obtain

$$
0 \leq \frac{F\left(x_{0}+\lambda\left(x_{1}-x_{0}\right)\right)-F\left(x_{0}\right)}{\lambda} \leq \frac{1}{\lambda} \int_{0}^{T}\left(p_{\lambda}\left(x_{0}+\lambda\left(x_{1}-x_{0}\right)\right)-p_{\lambda}\left(x_{0}\right)\right) u \mathrm{~d} t,
$$


where $p_{\lambda}$ is the solution to equation (53) with $p_{T}^{\lambda}=\theta\left|y_{\lambda}(T)-y_{d}\right|^{\theta-2}\left(y_{\lambda}(T)-y_{d}\right)$. We conclude with Lemma 4.2 by passing to the limit in the above inequality.

\section{REFERENCES}

[1] R.A. Adams, Sobolev spaces. Academic Press, New-York (1975).

[2] H. Amann, Dual semigroups and second order linear elliptic boundary value problems. Israel J. Math. 45 (1983) $225-254$.

[3] S. Anita, Optimal control of parameter distributed systems with impulses. Appl. Math. Optim. 29 (1994) 93-107.

[4] M. Berggren, R. Glowinski and J.L. Lions, A Computational Approach to Controllability Issues for Flow-Related Models, Part 1. Int. J. Comput. Fluid Dyn. 7 (1996) 237-252.

[5] M. Berggren, R. Glowinski and J.L. Lions, A Computational Approach to Controllability Issues for Flow-Related Models, Part 2. Int. J. Comput. Fluid Dyn. 6 (1996) 253-247.

[6] E. Casas, J.-P. Raymond and H. Zidani, Pontryagin's principle for local solutions of control problems with mixed control-state constraints. SIAM J. Control Optim. 39 (2000) 1182-1203.

[7] E. Casas, M. Mateos and J.-P. Raymond, Pontryagin's principle for the control of parabolic equations with gradient state constraints. Nonlinear Anal. (to appear).

[8] E.J. Dean and P. Gubernatis, Pointwise Control of Burgers' Equation - A Numerical Approach. Comput. Math. Appl. 22 (1991) 93-100.

[9] Z. Ding, L. Ji and J. Zhou, Constrained LQR Problems in Elliptic distributed Control systems with Point observations. SIAM 34 (1996) 264-294.

[10] J. Droniou and J.-P. Raymond, Optimal pointwise control of semilinear parabolic equations. Nonlinear Anal. 39 (2000) $135-156$.

[11] J.W. He and R. Glowinski, Neumann control of unstable parabolic systems: Numerical approach. J. Optim. Theory Appl. 96 (1998) 1-55.

[12] J.W. He, R. Glowinski, R. Metacalfe and J. Periaux, A numerical approach to the control and stabilization of advectiondiffusion systems: Application to viscous drag reduction, Flow control and optimization. Int. J. Comput. Fluid Dyn. 11 (1998) 131-156.

[13] H. Henrot and J. Sokolowski, Shape Optimization Problem for Heat Equation. Rapport de recherche INRIA (1997).

[14] D. Henry, Geometric Theory of Semilinear Parabolic Equations. Springer-Verlag, Berlin/Heidelberg/New-York (1981).

[15] K.-H. Hoffmann and J. Sokolowski, Interface optimization problems for parabolic equations. Control Cybernet. 23 (1994) 445-451.

[16] J.-P. Kernevez, The sentinel method and its application to environmental pollution problems. CRC Press, Boca Raton (1997).

[17] J.-L. Lions, Pointwise control for distributed systems, in Control and estimation in distributed parameters sytems, edited by H.T. Banks. SIAM, Philadelphia (1992) 1-39.

[18] O.A. Ladyzenskaja, V.A. Solonnikov and N.N. Ural'ceva, Linear and quasilinear equations of parabolic type. AMS, Providence, RI, Transl. Math. Monographs 23 (1968).

[19] H.-C. Lee and O.Yu. Imanuvilov, Analysis of Neumann boundary optimal control problems for the stationary Boussinesq equations including solid media. SIAM J. Control Optim. 39 (2000) 457-477.

[20] P.A. Nguyen, Optimal Control Localized on Thin Structure for Semilinear Parabolic Equations and the Boussinesq system. Thesis, Toulouse (2000).

[21] P.A. Nguyen and J.-P. Raymond, Control Localized On Thin Structure For Semilinear Parabolic Equations. Sém. Inst. H. Poincaré (to appear).

[22] A. Pazy, Semigroups of Linear Operators and Applications to Partial Differential Equations. Springer-Verlag, Berlin/Heidelberg/New-York (1983).

[23] M. Reed and B. Simon, Methods of Modern Mathematical Physics, Tome 2, Fourier Analysis, Self-Adjointness. Academic Press, Inc. (1975).

[24] J.-P. Raymond and H. Zidani, Hamiltonian Pontryagin's principles for control problems governed by semilinear parabolic equations. Appl. Math. Optim. 39 (1999) 143-177.

[25] J. Simon, Compact Sets in the Space $L^{p}(0, T ; B)$. Ann. Mat. Pura Appl. 196 (1987) 65-96.

[26] H. Triebel, Interpolation Theory, Functions Spaces, Differential Operators. North Holland Publishing Campany, Amsterdam/New-York/Oxford (1977).

[27] V. Vespri, Analytic Semigroups Generated in $H^{-m, p}$ by Elliptic Variational Operators and Applications to Linear Cauchy Problems, Semigroup theory and applications, edited by Clemens et al. Marcel Dekker, New-York (1989) 419-431. 